\title{
Article \\ Comparison of Potent Odorants in Traditional and Modern Types of Chinese Xiaoqu Liquor (Baijiu) Based on Odor Activity Values and Multivariate Analyses
}

\author{
Shuang Chen ${ }^{1,2} \mathbb{D}^{D}$, Jie Tang ${ }^{3} \mathbb{D}$, Shanshan Fan ${ }^{1}$, Jun Zhang ${ }^{1}$, Shenxi Chen ${ }^{3}$, Yuancai Liu ${ }^{3}$, Qiang Yang ${ }^{3, *}$ \\ and Yan $\mathrm{Xu}{ }^{1,2, *}$
}

1 Laboratory of Brewing Microbiology and Applied Enzymology, Key Laboratory of Industrial Biotechnology of Ministry of Education, State Key Laboratory of Food Science and Technology, School of Biotechnology, Jiangnan University, Wuxi 214122, China; shuangchen@jiangnan.edu.cn (S.C.); 6180201007@stu.jiangnan.edu.cn (S.F.); zjchem163@163.com (J.Z.)

2 Key Laboratory of Baijiu Supervision Technology for State Market Regulation, Chengdu 610097, China

3 Hubei Provincial Key Laboratory for Quality and Safety of Traditional Chinese Medicine Health Food, Jing Brand Research Institute, Jing Brand Co., Ltd., Daye 435100, China; tjjnan@163.com (J.T.); chenshenxi2006@163.com (S.C.); lyc@jingpai.com (Y.L.)

* $\quad$ Correspondence: yq@jingpai.com (Q.Y.); yxu@jiangnan.edu.cn (Y.X.); Tel.: +86-510-85918201 (Y.X.)

check for updates

Citation: Chen, S.; Tang, J.; Fan, S.; Zhang, J.; Chen, S.; Liu, Y.; Yang, Q.; $\mathrm{Xu}, \mathrm{Y}$. Comparison of Potent Odorants in Traditional and Modern Types of Chinese Xiaoqu Liquor (Baijiu) Based on Odor Activity Values and Multivariate Analyses. Foods 2021, 10, 2392. https:// doi.org/10.3390/foods10102392

Academic Editor: Amparo Gamero

Received: 5 September 2021

Accepted: 29 September 2021

Published: 9 October 2021

Publisher's Note: MDPI stays neutral with regard to jurisdictional claims in published maps and institutional affiliations.

Copyright: (C) 2021 by the authors. Licensee MDPI, Basel, Switzerland. This article is an open access article distributed under the terms and conditions of the Creative Commons Attribution (CC BY) license (https:// creativecommons.org/licenses/by/ $4.0 /)$.
Abstract: Predominant odorants in modern and traditional types of Chinese xiaoqu liquor (Baijiu) were identified and compared by the combined use of gas chromatography-olfactometry, odor activity values (OAVs), and multivariate analyses. A total of 79 aroma compounds were identified in a typical modern type xiaoqu Baijiu (M) and a typical traditional type xiaoqu Baijiu (T), 42 of them had $\mathrm{OAV}>1$ in both $\mathrm{M}$ and $\mathrm{T}$ samples. The main differences between the two samples were obtained for the concentration of 23 aroma-active compounds. A total of 22 samples made by different brewing processes were analyzed to confirm the differences. Partial least squares discriminant analysis confirmed that 20 compounds could be used as potential markers for discrimination between modern type xiaoqu Baijiu and traditional type xiaoqu Baijiu. Their difference in content is between 1.5 and 17.9 times for modern type xiaoqu Baijiu and traditional type xiaoqu Baijiu. The results showed the aroma characteristics of modern and traditional type xiaoqu Baijiu clearly and comprehensively, which will provide guidance for modern Baijiu quality control and evaluation.

Keywords: Chinese xiaoqu Baijiu; GC-O; GC-MS; odor activity values (OAVs); multivariate analyses

\section{Introduction}

Traditional fermented foods and beverages (TFFB) have been an important part of the human diet since the beginning of civilization [1]. TFFB remain widely favored by people because of their unique flavor and high nutritional value [2]. TFFB are primarily produced through largely uncontrolled spontaneous inoculation methods [3]. However, many drawbacks in traditional fermentation are directly or indirectly caused by a lack of control. These include low production efficiency, lack of consistency in product quality, and lengthy fermentation times. Thus, modern industrial development is essential in TFFB. Traditional fermentation process evolution and modernization have succeeded in many TFFB, such as soy sauce in the East [4] and cheese in the West [5].

Baijiu, Chinese liquor, is one of the most popular TFFB in China [6], which has thousands of years of history [7]. The annual production of Baijiu reached 7.41 billion liters and the sales revenue of Baijiu reached about 90 billion dollars in 2020, which played an important role in China's beverage industry [8]. The traditional fermentation and manufacturing methods of Baijiu have existed for centuries and strongly rely on individual operation skills and experience [6]. Traditional type Baijiu is produced using spontaneous fermentation 
processes in an open environment, and the fermentation temperature varies with the season. With the development of numerous technological innovations, the modern brewing technological process has been applied to Baijiu production, which not only improved the quality of Baijiu but also reduced energy consumption $[9,10]$. Mechanized operations are used in all stages of the brewing process and information technology is used to control the production environment in a relatively closed and stable environment, which maximally reduces the impact of the environment and operators during the brewing process.

There are very few publications comparing modern type and traditional type Baijiu. Wang et al. [11] separately studied the microbial succession and metabolism changes during Baijiu fermentation of traditional and modern workshops. The study showed that the microbial abundance of the traditional workshop is higher and the environmental microbiota have an important influence on the flavor of Baijiu. Gong et al. [12] explored the differences of volatile and nonvolatile compounds between traditional and mechanical raw Baijiu of sesame-like aroma-type Baijiu. The result showed that except for acids, phenols, and lactones, the total concentrations of the other compounds in traditional raw Baijiu were higher than those in mechanically produced raw Baijiu. Sun et al. [13] characterized 33 and 32 odor-active compounds in traditional and modern type xiaoqu Baijiu, respectively, by aroma extract dilution analysis and odor activity values (OAVs). However, the study was focused on only two samples and did not reveal the difference between the aroma compounds of the two types of xiaoqu Baijiu. The aroma is the most important characteristic of alcoholic beverages, and the production process is an important factor affecting the aroma profile [14]. So, the characterization of the aroma difference between traditional and modern-type Baijiu is of interest for improving modern brewing technology.

Xiaoqu Baijiu, one of the oldest and most popular Baijiu in China, is the first and most successful one to which the fully mechanized modern brewing process has been applied. Therefore, it is necessary to understand fully the similarities and differences of aromatic compounds in the Baijiu manufactured by different production processes. The primary objectives of this study were: (1) to identify the major aroma-active compounds in traditional and modern type xiaoqu Baijiu using gas chromatography-olfactometry (GC-O) and gas chromatography-mass spectrometry (GC-MS), (2) to confirm the contribution of those aroma compounds by quantitative analysis and their OAVs, and (3) to verify the aroma difference between the two types of xiaoqu Baijiu using multivariate statistical techniques.

\section{Materials and Methods}

\subsection{Baijiu Samples}

A total of 24 xiaoqu Baijiu raw liquors were analyzed in this study, including 12 modern type of xiaoqu Baijiu and 12 traditional type xiaoqu Baijiu. All samples were manufactured by Jing Brand Co., Ltd. (Daye, China) in 2019. The representative modern and traditional type Baijiu samples were chosen by the sensory panel composed of five national Chinese Baijiu tasting judges in Jing Brand Co., Ltd. Among the obtained xiaoqu Baijiu samples, one modern type xiaoqu Baijiu sample (M) and one traditional type xiaoqu Baijiu sample (T) were selected for liquid-liquid extraction (LLE) and GC-O analysis. All the samples were stored at $4{ }^{\circ} \mathrm{C}$ until analysis. The brand name is mentioned here for clear labeling, not for advertising purposes.

\subsection{Chemicals}

Chemical standards and internal standards (ISs) were supplied commercially at a high-purity grade (GC grade, $\geq 97 \%$ purity). Ethyl acetate, isobutyl acetate, isoamyl acetate, pentyl acetate, ethyl propanoate, ethyl 2-methylpropanoate, ethyl butanoate, ethyl 2-methylbutanoate, ethyl 3-methylbutanoate, 3-methylbutyl butanoate, ethyl pentanoate, ethyl hexanoate, propyl hexanoate, hexyl hexanoate, ethyl heptanoate, ethyl octanoate, ethyl nonanoate, ethyl decanoate, ethyl lactate, diethyl succinate, ethyl benzoate, ethyl phenylacetate, 2-phenethyl acetate, ethyl 3-phenylpropanoate, ethyl dodecanoate, ethyl tetradecanoate, 1-propanol, 2-methylpropanol, 1-butanol, 2-butanol, 3-methylbutanol, 1-pentanol, 
1-hexanol, 1-heptanol, 2-heptanol, 1-octanol, 3-octanol, 1-octen-3-ol, 1-nonanol, benzyl alcohol, $\beta$-phenethyl alcohol, 1,1-dimethoxyethane, 2-methylpropanal, 3-methylbutanal, hexanal, decanal, 1,1,3-triethoxypropane, benzaldehyde, benzeneacetaldehyde, 2-pentanone, 2octanone, acetophenone, 2-pentadecanone, acetic acid, propanoic acid, 2-methylpropanoic acid, butanoic acid, 3-methylbutanoic acid, pentanoic acid, 4-methylpentanoic acid, hexanoic acid, octanoic acid, guaiacol, 4-methylguaiacol, 4-ethylguaiacol, 4-vinylguaiacol, 4-methylphenol, 4-ethylphenol, linalool, $\beta$-damascenone, geraniol, geranylacetone, $\beta$ ionone, 2-pentylfuran, furfural, 2-furan methanol, $\gamma$-nonanolactone, dimethyl trisulfide, 2-thiophenecarboxaldehyde, 2-phenylethyl acetate- $\mathrm{D}_{3}$ (IS1), $n$-hexyl- $\mathrm{D}_{13}$ alcohol (IS2), 2,2dimethylpropanoic acid (IS3), 2-octanol (IS4), 2-methoxy-D D $_{3}$-phenol (IS5), and lactic acid were purchased from Sigma-Aldrich (Shanghai, China). A $\mathrm{C}_{6}-\mathrm{C}_{30} n$-alkanes mixture (Sigma-Aldrich, Shanghai, China) was used for the determination of retention indices (RIs). Sodium chloride $(\mathrm{NaCl})$, sodium carbonate $\left(\mathrm{Na}_{2} \mathrm{CO}_{3}\right)$, anhydrous sodium sulfate $\left(\mathrm{Na}_{2} \mathrm{SO}_{4}\right)$, and hydrochloric acid $(\mathrm{HCl})$ were purchased from China National Pharmaceutical Group Corp. (Shanghai, China). Ethanol (high-performance liquid chromatography (HPLC) grade) was purchased from J\&K Scientific Co., Ltd. (Beijing, China). Dichloromethane $\left(\mathrm{CH}_{2} \mathrm{Cl}_{2}\right.$, HPLC grade, ANPEL Scientific Instrument Co., Ltd., China) was distilled before use. Ultrapure water was obtained from a Milli-Q purification system (Millipore, Bedford, MA, USA).

\subsection{Extraction of Volatile Compounds}

The extraction method was modified from a previous study [15]. The $\mathrm{M}$ and $\mathrm{T}$ samples (100 mL each) were diluted to $10 \mathrm{vol} \%$ with boiled ultrapure water, saturated with $\mathrm{NaCl}$, and then extracted three times using freshly distilled $\mathrm{CH}_{2} \mathrm{Cl}_{2}(100 \mathrm{~mL}$ each time) in a separatory funnel. The combined extracts were further separated into acidic/water-soluble and neutral/basic fractions with the following method.

The combined extract (about $300 \mathrm{~mL}$ ) was washed three times with $\mathrm{Na}_{2} \mathrm{CO}_{3}(50 \mathrm{~mL}$ each time, $0.2 \mathrm{~mol} / \mathrm{L}, \mathrm{pH} 10.0$ ) and then washed with $30 \mathrm{~mL}$ of saturated $\mathrm{NaCl}$ solution. The obtained organic phase, containing the neutral/basic aroma compounds, was named the NBF fraction. The combined aqueous phase was acidified to $\mathrm{pH} 2.0$ with $\mathrm{HCl}(4.0 \mathrm{~mol} / \mathrm{L})$ and extracted three times with freshly distilled $\mathrm{CH}_{2} \mathrm{Cl}_{2}$ (50 mL each time). The combined extract (about $150 \mathrm{~mL}$ ), containing the remaining acidic aroma compounds, was named the AF fraction. Afterward, each fraction was dried with anhydrous $\mathrm{Na}_{2} \mathrm{SO}_{4}$ overnight and concentrated to a final volume of $0.5 \mathrm{~mL}$ under a gentle stream of nitrogen. These concentrated NBF and AF were stored at $-20{ }^{\circ} \mathrm{C}$ before the $\mathrm{GC}-\mathrm{O}$ analysis.

\subsection{Identification of Aroma Compounds Using GC-MS and GC-O}

GC-MS and GC-O analyses were performed on an Agilent 6890N GC equipped with an Agilent 5975 mass selective detector and a sniffing port (ODP 2, Gerstel, Württemberg, Germany). The sample was analyzed on both a DB-FFAP column $(60 \mathrm{~m} \times 0.25 \mathrm{~mm}$ i.d.; $0.25 \mu \mathrm{m}$ film thickness, Agilent Technologies, Inc., Santa Clara, CA,) and a DB-5 column (30 m $\times 0.25 \mathrm{~mm}$ i.d.; $0.25 \mu \mathrm{m}$ film thickness, Agilent Technologies, Inc.). High-purity helium (>99.999\%) was used as the carrier gas at a constant flow rate of $2 \mathrm{~mL} / \mathrm{min}$. The concentrated extract $(1 \mu \mathrm{L})$ was injected into the GC injector in splitless mode. The GC injector temperature was $250^{\circ} \mathrm{C}$. The oven temperature was held at $50^{\circ} \mathrm{C}$ for $2 \mathrm{~min}$, then raised to $230^{\circ} \mathrm{C}$ at a rate of $6{ }^{\circ} \mathrm{C} / \mathrm{min}$ and held for $15 \mathrm{~min}$. The mass spectrometer (MS) was operated in electron ionization mode at $70 \mathrm{eV}$. The time of solvent delay was $5 \mathrm{~min}$, and the temperature of the ion source was $230^{\circ} \mathrm{C}$. The mass range was set from 35 to $350 \mathrm{amu}$ in full-scan mode.

Four well-trained panelists (two females and two males, 22 years old on average) from the Laboratory of Brewing Microbiology and Applied Enzymology at Jiangnan University were selected for the GC-O study. During a GC run described above, a panelist placed his/her nose close to the sniffing port, responded to the aroma intensity of the stimulus, and recorded the retention time, descriptors, and intensities of the odor peak for each 
compound. The Osme values reflecting the aromatic intensity of the stimulus based on a six-point scale ranging from 0 to 5 was used for intensity judgment: $0=$ none, $1=$ very weak, 2 = weak, 3 = moderate, $4=$ strong, and $5=$ very strong. Every sample was sniffed twice by each panelist, if the aroma was detected six or more times on each column, an odor location was identified. The Osme value for aroma intensity was an average result of the four panelists.

The aroma compounds of the odor location were identified by comparing the aroma, mass spectrum (MS), and RIs to the pure standards (S). RIs were calculated based on the linear retention times of the $n$-alkanes $\left(C_{6}-C_{30}\right)$ in both the DB-FFAP and DB- 5 columns under the same chromatographic conditions.

\subsection{Quantitation of Aroma-Active Compounds by HS-SPME-Arrow Combined with GC-MS}

The aroma compounds in all xiaoqu Baijiu samples were quantitated using headspace solid-phase microextraction arrow (HS-SPME-Arrow) combined with GC-MS, as described previously [16]. An SPME-Arrow automatic headspace sampling system (CTC Analytics AG, Switzerland) with a $120 \mu \mathrm{m}$ divinylbenzene/carbon wide range/polydimethylsiloxane (DVB/CAR WR/PDMS) fiber (CTC Analytics AG, Basel, Switzerland) was used for extraction and injection. A total of $5 \mathrm{~mL}$ of the diluted $x i a o q u$ Baijiu sample $(10 \mathrm{vol} \%)$ was transferred into a $20 \mathrm{~mL}$ headspace glass vial, saturated with $1.5 \mathrm{~g} \mathrm{NaCl}$, and spiked with $40 \mu \mathrm{L}$ mixed ISs (final concentration: IS1, $146 \mu \mathrm{g} / \mathrm{L}$; IS2, $323 \mu \mathrm{g} / \mathrm{L}$; IS3, $1197 \mu \mathrm{g} / \mathrm{L}$; IS4, $294 \mu \mathrm{g} / \mathrm{L}$; IS5, $307 \mu \mathrm{g} / \mathrm{L})$. Tightly capped with a Teflon-faced silicone septum, the sample was equilibrated at $45{ }^{\circ} \mathrm{C}$ for $5 \mathrm{~min}$ and extracted for $45 \mathrm{~min}$ at the same temperature under stirring of $250 \mathrm{rpm}$. After extraction, the fiber was immediately inserted into the injection port of the GC for desorption at $250{ }^{\circ} \mathrm{C}$ for $5 \mathrm{~min}$. The samples were separated on a DB-FFAP column with splitless mode. The oven temperature was held at $40{ }^{\circ} \mathrm{C}$ for $2 \mathrm{~min}$, increased at $4^{\circ} \mathrm{C} / \mathrm{min}$ to $150{ }^{\circ} \mathrm{C}$, then held for $2 \mathrm{~min}$, raised at $6^{\circ} \mathrm{C} / \mathrm{min}$ to $230{ }^{\circ} \mathrm{C}$ and held for $5 \mathrm{~min}$.

Every standard stock solution was prepared by dissolving a standard compound in the model synthetic solution (10\% alcohol by volume at $\mathrm{pH} 4.0)$ and then diluting to 10 different concentrations. The same amount of ISs as in the xiaoqu Baijiu samples was added to the standard solutions, and then the working standard solutions were extracted using HS-SPME-Arrow and detected using GC-MS with the same conditions as for sample analysis. The standard curves were created by plotting the ratio of the peak area of the reference compounds to the corresponding IS against their concentration ratio. The analytical limit of detection (LOD) of aroma compounds was obtained from the lowest concentrations of the analyte standard solutions based on a signal-to-noise ratio of 3 . The limits of quantitation (LOQ) was defined as the lowest concentration of the calibration curve based on a signal-to-noise ratio of 10. The accuracy is reported as the percentage recovery of a known amount of target analyte added to the xiaoqu Baijiu sample. All analyses were performed in triplicate.

\subsection{Statistical Analysis}

Principal component analysis (PCA) was performed to explore the clustering of the xiaoqu Baijiu samples in terms of their aroma compounds. To maximize the separation among samples and to identify the aroma compounds responsible for the separation, partial least squares discriminant analysis (PLS-DA) was used. PCA and PLS-DA were conducted using SIMCA version 14 software (Umetrics, Umearing, Sweden). The heatmap and hierarchical clustering analysis (HCA) were performed using HemI 1.0 (The CUCKOO Workgroup, http:/ / hemi.biocuckoo.org, accessed on 4 September 2021).

\section{Results and Discussion}

\subsection{Identification of Aroma Compounds in Xiaoqu Baijiu Using GC-O}

By extraction with $\mathrm{CH}_{2} \mathrm{Cl}_{2}$, an organic phase was obtained that exactly showed the typical xiaoqu Baijiu aroma when sniffed on a filter paper by the panelists. Thus, it was 
appropriate and reliable to be used to analyze the profile of aroma compounds in $x i a o q u$ Baijiu. To facilitate GC-O analysis and compound identification, the extract obtained was further separated into AF and NBF fractions to reduce complexity as previously described [15]. To identify the constituents responsible for the odors, aroma areas were first located using GC-O. The RI of each odorant regions on the DB-FFAP and DB-5 columns was calculated. Then, the aroma quality and odor descriptor of each odorant were determined by comparison to a Chinese Baijiu flavor database (an in-house database consisting of more than 900 odor-active standard compounds). The identities of the tentatively assigned aroma compounds were finally confirmed by comparing their MS data (EI mode) with those of the standard reference compounds.

As shown in Table 1, a total of 79 odorants were located in the $\mathrm{M}$ and $\mathrm{T}$ samples in this study. The results showed that both liquors had a similar aroma profile, but the Osme values varied (Figure 1). Most of these components are well-known odorants in Baijiu $[7,17,18]$, but 36 of them have not been reported in a previous study of xiaoqu Baijiu [13]. Among the 79 aroma compounds, 26 esters, 15 alcohols, 12 aldehydes and ketones, nine acids, six phenols, five terpenoids, four furans, and two sulfur compounds were detected in the $\mathrm{M}$ and $\mathrm{T}$ samples.

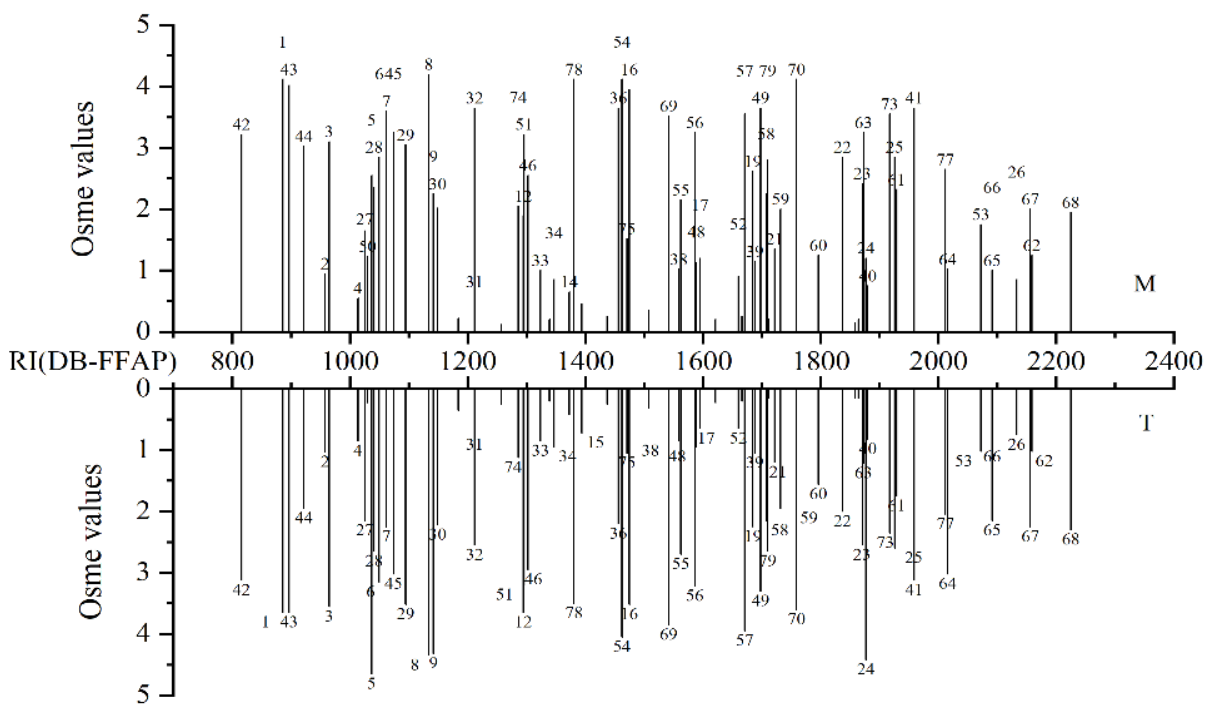

Figure 1. Osme values of aroma extract from $\mathrm{M}$ and $\mathrm{T}$ samples by GC-O and GC-MS analyses (Annotation: Number on peak corresponds to Table 2, the abscissa represents the retention index of the compounds on the DB-FFAP chromatographic column, and the ordinate represents the aroma intensity of the compounds.).

The dominant group of odorants identified was esters, particularly ethyl esters. The most important esters identified were ethyl acetate, isoamyl acetate, ethyl 2-methylpropanoate, and ethyl octanoate with Osme values above 3.00 in both samples, contributing a strong fruity note. In general, the T sample had higher Osme values for most esters than the M sample, such as ethyl butanoate (Osme values $=2.55 ; 4.65)$, ethyl pentanoate (Osme values $=2.25$; 4.32 ), ethyl hexanoate (Osme values $=1.90 ; 3.65)$, and ethyl 3-phenylpropanoate (Osme values $=1.20 ; 4.42$ ). However, some other esters had higher Osme values in the M sample. They were ethyl acetate $($ Osme values $=4.12 ; 3.65)$, ethyl 3-methylbutanoate (Osme values = 3.60; 2.25 ), ethyl octanoate (Osme values $=3.95 ; 3.52)$, ethyl decanoate (Osme values $=2.62 ; 2.25$ ), and ethyl phenylacetate (Osme values $=2.85 ; 2.00$ ).

The second dominant group of aroma compounds identified were alcohols. Among the alcoholic compounds identified in the M sample, 3-methylbutanol, 1-octen-3-ol, and $\beta$-phenethyl alcohol showed the highest Osme values of 3.65, contributing whiskey, mushroom, and honey odors, respectively. However, 2-methylpropanol with the Osme value of 3.52 was the most important aroma contributor in the T sample. 
Table 1. Aroma compounds in Chinese xiaoqu Baijiu identified by gas chromatography-olfactometry (GC-O) and gas chromatography-mass spectrometry (GC-MS).

\begin{tabular}{|c|c|c|c|c|c|c|c|c|}
\hline \multirow{2}{*}{ No. } & \multirow{2}{*}{ Compound } & \multicolumn{2}{|c|}{$\mathbf{R I}^{\mathrm{a}}$} & \multirow[t]{2}{*}{ Odor $^{b}$} & \multirow[t]{2}{*}{ Fraction $^{c}$} & \multirow{2}{*}{$\begin{array}{c}\text { Identification }^{\text {Mean }^{\mathrm{d}}} \\
\end{array}$} & \multicolumn{2}{|c|}{$\begin{array}{l}\text { Osme } \\
\text { Values }\end{array}$} \\
\hline & & DB-FFAP & DB-5 & & & & $\mathbf{M}$ & $\mathrm{T}$ \\
\hline & Esters & & & & & & & \\
\hline 1 & Ethyl acetate & 886 & 589 & Sweet, fruity & $\mathrm{AF}+\mathrm{NBF}$ & RI, aroma, S & 4.12 & 3.65 \\
\hline 2 & Ethyl propionate & 958 & 695 & Fruity & $\mathrm{AF}+\mathrm{NBF}$ & MS, RI, aroma, S & 0.95 & 1.03 \\
\hline 3 & $\begin{array}{c}\text { Ethyl } \\
\text { 2-methylpropanoate }\end{array}$ & 965 & 752 & Nail polish & NBF & MS, RI, aroma, S & 3.10 & 3.55 \\
\hline 4 & Isobutyl acetate & 1014 & 768 & Fruity & NBF & MS, RI, aroma, S & 0.55 & 0.85 \\
\hline 5 & Ethyl butanoate & 1037 & 817 & Sweet, fruity & NBF & MS, RI, aroma, S & 2.55 & 4.65 \\
\hline 6 & Ethyl 2-methylbutanoate & 1050 & 841 & Sweet, fruity & NBF & MS, RI, aroma, S & 2.85 & 3.15 \\
\hline 7 & Ethyl 3-methylbutanoate & 1062 & 856 & Sweet, fruity & NBF & MS, RI, aroma, S & 3.60 & 2.25 \\
\hline 8 & Isoamyl acetate & 1134 & 878 & Banana & NBF & MS, RI, aroma, S & 4.20 & 4.35 \\
\hline 9 & Ethyl pentanoate & 1142 & 903 & Fruity & $\mathrm{AF}+\mathrm{NBF}$ & MS, RI, aroma, S & 2.25 & 4.32 \\
\hline 10 & Pentyl acetate & 1184 & 926 & Banana & NBF & MS, RI, aroma, S & 0.22 & 0.35 \\
\hline 11 & 3-Methylbutyl butanoate & 1257 & 1061 & Fruity & NBF & MS, RI, aroma, S & 0.12 & 0.25 \\
\hline 12 & Ethyl hexanoate & 1295 & 1025 & Fruity & $\mathrm{AF}+\mathrm{NBF}$ & MS, RI, aroma, S & 1.90 & 3.65 \\
\hline 13 & Propyl hexanoate & 1339 & 1081 & Fruity & $\mathrm{AF}+\mathrm{NBF}$ & MS, RI, aroma, S & 0.20 & 0.20 \\
\hline 14 & Ethyl lactate & 1373 & 825 & Fruity & $\mathrm{AF}+\mathrm{NBF}$ & MS, RI, aroma, S & 0.65 & 0.42 \\
\hline 15 & Ethyl heptanoate & 1394 & 1113 & Fruity & NBF & MS, RI, aroma, S & 0.45 & 0.72 \\
\hline 16 & Ethyl octanoate & 1475 & 1200 & Fruity & $\mathrm{AF}+\mathrm{NBF}$ & MS, RI, aroma, S & 3.95 & 3.52 \\
\hline 17 & Ethyl nonanoate & 1595 & 1284 & Fruity & NBF & MS, RI, aroma, S & 1.20 & 0.65 \\
\hline 18 & Hexyl hexanoate & 1621 & 1381 & Fruity & NBF & MS, RI, aroma, S & 0.20 & 0.22 \\
\hline 19 & Ethyl decanoate & 1685 & 1391 & Grape & NBF & MS, RI, aroma, S & 2.62 & 2.25 \\
\hline 20 & Diethyl succinate & 1711 & 1179 & Sweet & $\mathrm{AF}+\mathrm{NBF}$ & MS, RI, aroma, S & 0.20 & 0.15 \\
\hline 21 & Ethyl benzoate & 1722 & 1165 & Fruity & NBF & MS, RI, aroma, S & 1.35 & 1.20 \\
\hline 22 & Ethyl phenylacetate & 1837 & 1254 & Fruity & NBF & MS, RI, aroma, S & 2.85 & 2.00 \\
\hline 23 & 2-Phenethyl acetate & 1871 & 1271 & Rose & NBF & MS, RI, aroma, S & 2.42 & 2.55 \\
\hline 24 & $\begin{array}{c}\text { Ethyl } \\
\text { 3-phenylpropanoate }\end{array}$ & 1877 & 1345 & Floral & NBF & MS, RI, aroma, S & 1.20 & 4.42 \\
\hline 25 & Ethyl dodecanoate & 1926 & 1581 & Fruity & NBF & MS, RI, aroma, S & 2.85 & 2.60 \\
\hline 26 & $\begin{array}{c}\text { Ethyl tetradecanoate } \\
\text { Alcohols }\end{array}$ & 2133 & 1790 & Coconut & NBF & MS, RI, aroma, S & 0.85 & 0.75 \\
\hline 27 & 2-Butanol & 1025 & - & Wine & NBF & MS, RI, aroma, S & 1.65 & 2.15 \\
\hline 28 & 1-Propanol & 1041 & - & Alcoholic & $\mathrm{AF}+\mathrm{NBF}$ & MS, RI, aroma, S & 2.35 & 2.65 \\
\hline 29 & 2-Methylpropanol & 1095 & 620 & Solvent & $\mathrm{AF}+\mathrm{NBF}$ & MS, RI, aroma, S & 3.05 & 3.52 \\
\hline 30 & 1-Butanol & 1149 & 669 & Fruity & $\mathrm{AF}+\mathrm{NBF}$ & MS, RI, aroma, S & 2.02 & 2.22 \\
\hline 31 & 1-Pentanol & 1212 & 761 & Fruity & NBF & MS, RI, aroma, S & 0.65 & 0.75 \\
\hline 32 & 3-Methylbutanol & 1212 & 790 & Whisky, burnt & $\mathrm{AF}+\mathrm{NBF}$ & MS, RI, aroma, S & 3.65 & 2.55 \\
\hline 33 & 2-Heptanol & 1324 & 901 & Mushroom & NBF & MS, RI, aroma, S & 1.00 & 0.85 \\
\hline 34 & 1-Hexanol & 1347 & 892 & Fruity & NBF & MS, RI, aroma, S & 0.85 & 0.95 \\
\hline 35 & 3-Octanol & 1437 & 992 & Mushroom & NBF & MS, RI, aroma, S & 0.25 & 0.25 \\
\hline 36 & 1-Octen-3-ol & 1456 & 964 & Mushroom & NBF & MS, RI, aroma, S & 3.65 & 2.20 \\
\hline 37 & 1-Heptanol & 1462 & 970 & Green & NBF & MS, RI, aroma, S & 0.20 & 0.10 \\
\hline 38 & 1-Octanol & 1559 & 1089 & Fruity & NBF & MS, RI, aroma, S & 1.02 & 0.85 \\
\hline 39 & 1-Nonanol & 1688 & 1168 & Grass & NBF & MS, RI, aroma, S & 1.15 & 1.05 \\
\hline 40 & Benzyl alcohol & 1880 & 1033 & Rose & NBF & MS, RI, aroma, S & 0.75 & 0.82 \\
\hline 41 & $\begin{array}{c}\beta \text {-Phenethyl alcohol } \\
\text { Aldehydes }\end{array}$ & 1959 & 1130 & Rose, honey & $\mathrm{AF}+\mathrm{NBF}$ & MS, RI, aroma, S & 3.65 & 3.12 \\
\hline 42 & 2-Methyl propanal & 816 & 550 & Malty & NBF & RI, aroma, S & 3.22 & 3.12 \\
\hline 43 & 1,1-Dimethoxyethane & 897 & 730 & Fruity & NBF & MS, RI, aroma, S & 4.02 & 3.65 \\
\hline 44 & 3-Methylbutanal & 922 & 621 & Malty & NBF & MS, RI, aroma, S & 3.03 & 1.95 \\
\hline 45 & Hexanal & 1074 & 803 & Grassy, green & NBF & MS, RI, aroma, S & 3.25 & 3.02 \\
\hline 46 & 1,1,3-Triethoxypropane & 1303 & 1128 & Fruity & $\mathrm{AF}+\mathrm{NBF}$ & MS, RI, aroma, S & 2.55 & 2.95 \\
\hline 47 & Decanal & 1508 & 1228 & Orange & NBF & MS, RI, aroma, S & 0.35 & 0.32 \\
\hline 48 & Benzaldehyde & 1589 & 975 & Almond & NBF & MS, RI, aroma, S & 1.12 & 0.95 \\
\hline 49 & $\begin{array}{c}\text { Benzeneacetaldehyde } \\
\text { Ketones }\end{array}$ & 1698 & 1044 & Honey & NBF & MS, RI, aroma, S & 3.65 & 3.30 \\
\hline 50 & 2-Pentanone & 1030 & - & Fruity & NBF & MS, RI, aroma, S & 1.23 & 0.22 \\
\hline 51 & 2-Octanone & 1296 & 997 & Soap & NBF & MS, RI, aroma, S & 3.22 & 3.20 \\
\hline 52 & Acetophenone & 1660 & 1076 & Floral & NBF & MS, RI, aroma, S & 0.90 & 0.65 \\
\hline 53 & 2-Pentadecanone & 2072 & - & Fruity & NBF & MS, RI, aroma, S & 1.75 & 1.02 \\
\hline
\end{tabular}


Table 1. Cont

\begin{tabular}{|c|c|c|c|c|c|c|c|c|}
\hline \multirow{2}{*}{ No. } & \multirow[t]{2}{*}{ Compound } & \multicolumn{2}{|c|}{$\mathbf{R I}^{\mathrm{a}}$} & \multirow[t]{2}{*}{ Odor $^{b}$} & \multirow[t]{2}{*}{ Fraction $^{c}$} & \multirow{2}{*}{$\begin{array}{c}\text { Identification } \\
\text { Mean }^{\mathrm{d}}\end{array}$} & \multicolumn{2}{|c|}{$\begin{array}{l}\text { Osme } \\
\text { Values }\end{array}$} \\
\hline & & DB-FFAP & DB-5 & & & & $\mathbf{M}$ & $\mathbf{T}$ \\
\hline & Acids & & & & & & & \\
\hline 54 & Acetic acid & 1462 & 606 & Vinegar & $\mathrm{AF}$ & MS, RI, aroma, S & 4.12 & 4.05 \\
\hline 55 & Propanoic acid & 1562 & - & Rancid & $\mathrm{AF}$ & MS, RI, aroma, S & 2.15 & 2.70 \\
\hline 56 & 2-Methylpropanoic acid & 1586 & 790 & Sweaty & $\mathrm{AF}$ & MS, RI, aroma, S & 3.25 & 3.22 \\
\hline 57 & Butanoic acid & 1671 & 800 & Sweaty & $\mathrm{AF}$ & MS, RI, aroma, S & 3.55 & 3.95 \\
\hline 58 & 3-Methylbutanoic acid & 1707 & 835 & Sweaty & $\mathrm{AF}$ & MS, RI, aroma, S & 2.25 & 2.15 \\
\hline 59 & Pentanoic acid & 1732 & - & Sweat & $\mathrm{AF}$ & MS, RI, aroma, S & 2.00 & 1.95 \\
\hline 60 & 4-Methylpentanoic acid & 1796 & - & Rancid & $\mathrm{AF}$ & MS, RI, aroma, S & 1.25 & 1.56 \\
\hline 61 & Hexanoic acid & 1929 & 971 & Sweaty & $\mathrm{AF}$ & MS, RI, aroma, S & 2.32 & 1.75 \\
\hline 62 & $\begin{array}{l}\text { Octanoic acid } \\
\text { Phenols }\end{array}$ & 2159 & 1280 & Sweaty & $\mathrm{AF}$ & MS, RI, aroma, S & 1.25 & 1.02 \\
\hline 63 & Guaiacol & 1874 & 1090 & Smoky & NBF & MS, RI, aroma, S & 3.25 & 1.22 \\
\hline 64 & 4-Methylguaiacol & 2016 & 1199 & Smoky & $\mathrm{AF}+\mathrm{NBF}$ & MS, RI, aroma, S & 1.02 & 3.02 \\
\hline 65 & 4-Ethylguaiacol & 2091 & 1297 & Spice & $\mathrm{AF}+\mathrm{NBF}$ & MS, RI, aroma, S & 0.95 & 2.15 \\
\hline 66 & 4-Methyl phenol & 2091 & - & Medicinal & $\mathrm{AF}+\mathrm{NBF}$ & MS, RI, aroma, S & 1.00 & 0.95 \\
\hline 67 & 4-Vinylguaiacol & 2156 & 1311 & Smoky & NBF & MS, RI, aroma, S & 2.00 & 2.25 \\
\hline 68 & $\begin{array}{l}\text { 4-Ethyl phenol } \\
\text { Terpenoids }\end{array}$ & 2225 & 1172 & Smoky & NBF & MS, RI, aroma, S & 1.95 & 2.30 \\
\hline 69 & Linalool & 1542 & 1099 & Floral & NBF & MS, RI, aroma, S & 3.52 & 3.85 \\
\hline 70 & $\beta$-Damascenone & 1759 & 1378 & Rose & NBF & MS, RI, aroma, S & 4.12 & 3.60 \\
\hline 71 & Geraniol & 1858 & 1277 & Rose & NBF & MS, RI, aroma, S & 0.15 & 0.15 \\
\hline 72 & Geranylacetone & 1864 & 1460 & Sweet & NBF & MS, RI, aroma, S & 0.20 & 0.15 \\
\hline 73 & $\begin{array}{c}\beta \text {-Ionone } \\
\text { Others }\end{array}$ & 1917 & 1477 & Floral & NBF & MS, RI, aroma, S & 3.55 & 2.35 \\
\hline 74 & 2-Pentylfuran & 1286 & - & Sweet, fruity & NBF & MS, RI, aroma, S & 2.05 & 1.12 \\
\hline 75 & Furfural & 1471 & 845 & Bread & NBF & MS, RI, aroma, S & 1.52 & 1.05 \\
\hline 76 & 2-Furan methanol & 1666 & 813 & Floral & NBF & MS, RI, aroma, S & 0.25 & 0.20 \\
\hline 77 & $\gamma$-Nonanolactone & 2012 & 1358 & Coconut & NBF & MS, RI, aroma, S & 2.65 & 2.05 \\
\hline 78 & Dimethyl trisulfide & 1381 & 976 & Cabbage & NBF & MS, RI, aroma, S & 4.12 & 3.50 \\
\hline 79 & Thiophenecarboxaldehyde & 1710 & - & Almond & NBF & MS, RI, aroma, S & 2.80 & 2.65 \\
\hline
\end{tabular}

${ }^{a}$ Retention indices determined by GC-MS on two different stationary phases (DB-FFAP and DB-5). ${ }^{\mathrm{b}}$ Odor quality perceived at the sniffing port. ${ }^{c}$ The odorants were detected in fraction AF, acidic/water-soluble fraction; NBF, neutral/basic fraction. ${ }^{\mathrm{d}}$ Identification based on RI (retention index), MS (mass spectrometry), aroma (odor description by comparison to the reference standards by GC-O), and S (standards).

Table 2. Quantitative methodological parameters of aroma compounds in Chinese xiaoqu Baijiu.

\begin{tabular}{|c|c|c|c|c|c|c|c|c|}
\hline No. & Compound & $\begin{array}{c}\text { Quantitative } \\
\text { Ion }(\mathbf{m} / \mathbf{z})\end{array}$ & IS $^{a}$ & Slope & Intercept & $\mathbf{R}^{2}$ & Recovery (\%) & $\mathrm{LOQ} / \mu \mathrm{g} / \mathrm{L}$ \\
\hline 1 & Ethyl acetate & 61 & IS1 & 0.0035 & -0.0531 & 0.9995 & 93.46 & 3039.61 \\
\hline 2 & Ethyl propionate & 57 & IS1 & 0.0880 & -3.5225 & 0.9973 & 91.32 & 721.15 \\
\hline 3 & $\begin{array}{c}\text { Ethyl } \\
\text { 2-methylpropanoate }\end{array}$ & 71 & IS1 & 0.3080 & -0.0356 & 0.9993 & 87.79 & 47.95 \\
\hline 4 & Isobutyl acetate & 43 & IS1 & 0.3226 & 0.7735 & 0.9924 & 98.85 & 78.19 \\
\hline 5 & Ethyl butanoate & 71 & IS1 & 0.1670 & 0.1325 & 0.9985 & 85.07 & 125.58 \\
\hline 6 & Ethyl 2-methylbutanoate & 57 & IS1 & 0.3005 & 0.0479 & 0.9999 & 95.76 & 74.54 \\
\hline 7 & Ethyl 3-methylbutanoate & 88 & IS1 & 0.4875 & 0.0071 & 0.9995 & 85.05 & 5.68 \\
\hline 8 & Isoamyl acetate & 70 & IS1 & 0.0775 & 0.3756 & 0.9985 & 109.34 & 80.05 \\
\hline 9 & Ethyl pentanoate & 88 & IS1 & 0.3010 & -0.1164 & 0.9992 & 82.74 & 26.68 \\
\hline 10 & Pentyl acetate & 70 & IS1 & 0.8020 & -0.0135 & 0.9953 & 82.54 & 2.13 \\
\hline 11 & 3-Methylbutyl butanoate & 71 & IS1 & 1.2970 & 0.0065 & 0.9963 & 105.76 & 0.38 \\
\hline 12 & Ethyl hexanoate & 88 & IS1 & 2.2610 & 1.5085 & 0.996 & 101.03 & 59.81 \\
\hline 13 & Propyl hexanoate & 99 & IS1 & 3.6525 & -0.1232 & 0.9934 & 94.93 & 2.10 \\
\hline 14 & Ethyl lactate & 45 & IS1 & 0.6550 & 0.0325 & 0.9945 & 90.94 & 1.17 \\
\hline 15 & Ethyl heptanoate & 88 & IS1 & 4.5020 & -0.0636 & 0.9933 & 93.04 & 9.80 \\
\hline 16 & Ethyl octanoate & 88 & IS1 & 1.1625 & 7.0776 & 0.9900 & 99.18 & 195.36 \\
\hline 17 & Ethyl nonanoate & 88 & IS1 & 5.5880 & 0.086 & 0.9965 & 88.20 & 3.41 \\
\hline 18 & Hexyl hexanoate & 117 & IS1 & 7.8150 & -0.0281 & 0.9927 & 88.08 & 0.60 \\
\hline 19 & Ethyl decanoate & 88 & IS1 & 11.147 & 0.1139 & 0.9997 & 98.65 & 236.84 \\
\hline 20 & Diethyl succinate & 101 & IS1 & 0.2750 & -0.8877 & 0.9975 & 105.78 & 73.66 \\
\hline 21 & Ethyl benzoate & 105 & IS1 & 5.7595 & 0.1188 & 0.9964 & 96.98 & 0.41 \\
\hline 22 & Ethyl phenylacetate & 91 & IS1 & 1.6570 & -0.2062 & 0.9967 & 112.18 & 39.07 \\
\hline 23 & 2-Phenethyl acetate & 104 & IS1 & 0.3597 & 0.0593 & 0.9996 & 96.49 & 7.89 \\
\hline
\end{tabular}


Table 2. Cont.

\begin{tabular}{|c|c|c|c|c|c|c|c|c|}
\hline No. & Compound & $\begin{array}{l}\text { Quantitative } \\
\text { Ion }(\mathrm{m} / \mathrm{z})\end{array}$ & IS $^{a}$ & Slope & Intercept & $\mathbf{R}^{2}$ & Recovery (\%) & $\mathrm{LOQ} / \mu \mathrm{g} / \mathrm{L}$ \\
\hline 24 & $\begin{array}{c}\text { Ethyl } \\
\text { 3-phenylpropanoate }\end{array}$ & 104 & IS1 & 2.2125 & 0.1824 & 0.9987 & 103.68 & 7.81 \\
\hline 25 & Ethyl dodecanoate & 88 & IS1 & 6.5970 & -0.2234 & 0.9978 & 83.70 & 4.05 \\
\hline 26 & Ethyl tetradecanoate & 88 & IS1 & 3.8505 & -0.2599 & 0.9963 & 89.39 & 3.91 \\
\hline 27 & 2-Butanol & 45 & IS2 & 0.0879 & 0.0581 & 0.9951 & 87.31 & 37.50 \\
\hline 28 & 1-Propanol & 59 & IS2 & 0.0044 & -0.1675 & 0.9995 & 84.21 & 7556.48 \\
\hline 29 & 2-Methylpropanol & 74 & IS2 & 0.0043 & -0.0098 & 0.9982 & 85.75 & 549.14 \\
\hline 30 & 1-Butanol & 56 & IS2 & 0.0781 & -0.4634 & 0.9952 & 105.22 & 363.80 \\
\hline 31 & 1-Pentanol & 55 & IS2 & 0.2384 & -0.0499 & 0.9955 & 103.65 & 15.62 \\
\hline 32 & 3-Methylbutanol & 55 & IS2 & 0.0389 & 3.3248 & 0.9989 & 101.61 & 2320.31 \\
\hline 33 & 2-Heptanol & 55 & IS2 & 0.8462 & 0.0115 & 0.9981 & 114.83 & 6.13 \\
\hline 34 & 1-Hexanol & 56 & IS2 & 0.4043 & 1.1356 & 0.9978 & 111.05 & 132.38 \\
\hline 35 & 3-Octanol & 59 & IS2 & 1.0170 & 0.0382 & 0.9987 & 113.69 & 9.62 \\
\hline 36 & 1-Octen-3-ol & 57 & IS2 & 8.5931 & 0.0952 & 0.9991 & 107.11 & 1.40 \\
\hline 37 & 1-Heptanol & 70 & IS2 & 2.8290 & -0.0062 & 0.9965 & 114.48 & 0.43 \\
\hline 38 & 1-Octanol & 56 & IS2 & 3.0323 & 0.6008 & 0.9973 & 86.28 & 5.67 \\
\hline 39 & 1-Nonanol & 56 & IS2 & 10.2200 & 0.0967 & 0.9983 & 90.01 & 0.71 \\
\hline 40 & Benzyl alcohol & 79 & IS2 & 0.1822 & 0.0033 & 0.9956 & 83.53 & 3.78 \\
\hline 41 & $\beta$-Phenethyl alcohol & 91 & IS2 & 0.4217 & 0.3523 & 0.9972 & 85.43 & 58.36 \\
\hline 42 & 2-Methyl propanal & 72 & IS4 & 0.0304 & -0.0592 & 0.9973 & 91.71 & 69.64 \\
\hline 43 & 1,1-Dimethoxyethane & 73 & IS4 & 0.0057 & -0.1715 & 0.9978 & 83.29 & 3096.02 \\
\hline 44 & 3-Methylbutanal & 58 & IS4 & 0.0364 & -0.015 & 0.9974 & 85.70 & 210.62 \\
\hline 45 & Hexanal & 56 & IS4 & 0.2427 & -0.0386 & 0.9973 & 104.62 & 8.10 \\
\hline 46 & 1,1,3-Triethoxypropane & 59 & IS4 & 0.0234 & -0.0125 & 0.9993 & 93.54 & 68.76 \\
\hline 47 & Decanal & 57 & IS4 & 3.5109 & -0.0329 & 0.9974 & 8734.5 & 0.45 \\
\hline 48 & Benzaldehyde & 106 & IS4 & 0.9092 & 0.0855 & 0.9959 & 118.17 & 8.15 \\
\hline 49 & Benzeneacetaldehyde & 91 & IS4 & 0.2074 & -0.0312 & 0.9997 & 87.34 & 19.95 \\
\hline 50 & 2-Pentanone & 86 & IS4 & 0.0542 & -0.0248 & 0.9996 & 87.77 & 27.50 \\
\hline 51 & 2-Octanone & 58 & IS4 & 3.8946 & -0.1028 & 0.9992 & 108.62 & 2.23 \\
\hline 52 & Acetophenone & 105 & IS4 & 2.0508 & 0.0256 & 0.9958 & 96.17 & 0.36 \\
\hline 53 & 2-Pentadecanone & 58 & IS4 & 63.259 & -0.0799 & 0.9963 & 81.80 & 0.20 \\
\hline 54 & Acetic acid & 60 & IS3 & 0.0082 & -0.0565 & 0.9996 & 108.72 & 8260.31 \\
\hline 55 & Propanoic acid & 74 & IS3 & 0.0235 & -0.0334 & 0.9956 & 104.88 & 2970.00 \\
\hline 56 & 2-Methylpropanoic acid & 73 & IS3 & 0.0851 & -0.0764 & 0.9963 & 88.10 & 742.16 \\
\hline 57 & Butanoic acid & 60 & IS3 & 0.1737 & -0.0698 & 0.9989 & 108.42 & 523.46 \\
\hline 58 & 3-Methylbutanoic acid & 60 & IS3 & 0.5851 & -0.0283 & 0.999 & 107.91 & 105.76 \\
\hline 59 & Pentanoic acid & 73 & IS3 & 0.2789 & -0.1994 & 0.9982 & 96.28 & 1166.94 \\
\hline 60 & 4-Methylpentanoic acid & 57 & IS3 & 1.1236 & -0.0169 & 0.9962 & 91.06 & 7.84 \\
\hline 61 & Hexanoic acid & 60 & IS3 & 2.1683 & 0.0657 & 0.9995 & 100.40 & 69.61 \\
\hline 62 & Octanoic acid & 73 & IS3 & 3.0605 & -0.0883 & 0.9987 & 99.70 & 21.70 \\
\hline 63 & Guaiacol & 109 & IS5 & 0.6820 & 0.0016 & 0.9993 & 96.06 & 1.83 \\
\hline 64 & 4-Methylguaiacol & 138 & IS5 & 1.1433 & -0.0054 & 0.9979 & 106.42 & 0.67 \\
\hline 65 & 4-Ethylguaiacol & 137 & IS5 & 3.1906 & -0.0012 & 0.9989 & 102.74 & 0.28 \\
\hline 66 & 4-Methyl phenol & 107 & IS5 & 0.8881 & -0.0029 & 0.9977 & 107.47 & 1.48 \\
\hline 67 & 4-Vinylguaiacol & 150 & IS5 & 0.3140 & -0.0226 & 0.9969 & 99.26 & 4.00 \\
\hline 68 & 4-Ethyl phenol & 107 & IS5 & 2.0922 & -0.0002 & 0.9982 & 104.70 & 0.07 \\
\hline 69 & Linalool & 71 & IS4 & 2.3080 & -0.0111 & 0.9999 & 98.18 & 1.57 \\
\hline 70 & $\beta$-Damascenone & 69 & IS4 & 0.0225 & 0.0001 & 0.9972 & 98.63 & 16.00 \\
\hline 71 & Geraniol & 69 & IS4 & 2.8196 & 0.0086 & 0.9962 & 92.21 & 0.08 \\
\hline 72 & Geranylacetone & 69 & IS4 & 12.5120 & 0.0091 & 0.9985 & 97.76 & 0.08 \\
\hline 73 & $\beta$-Ionone & 177 & IS4 & 5.8592 & 0.0075 & 0.9954 & 87.96 & 0.19 \\
\hline 74 & 2-Pentylfuran & 81 & IS4 & 3.8924 & -0.7053 & 0.9982 & 101.65 & 13.70 \\
\hline 75 & Furfural & 96 & IS4 & 0.0429 & 0.1024 & 0.9964 & 107.74 & 224.30 \\
\hline 76 & 2-Furan methanol & 98 & IS4 & 0.0064 & -0.0137 & 0.9980 & 83.96 & 120.40 \\
\hline 77 & $\gamma$-Nonanolactone & 85 & IS4 & 1.0395 & -0.0052 & 0.9980 & 86.55 & 0.36 \\
\hline 78 & Dimethyl trisulfide & 126 & IS4 & 0.4135 & 0.0003 & 0.9994 & 92.86 & 0.25 \\
\hline 79 & Thiophenecarboxaldehyde & 111 & IS4 & 0.0125 & 0.0002 & 0.9994 & 87.59 & 9.71 \\
\hline
\end{tabular}

a IS, internal standard; IS1, 2-phenylethyl acetate-D ${ }_{3}$; IS2, n-hexyl-D 13 alcohol; IS3, 2,2-dimethyl-propanoic acid; IS4, 2-octanol; IS5, 2-methoxy-D - -phenol.

Based on GC-O and GC-MS, eight aroma aldehydes were detected. Aromatic aldehydes seemed to be important in xiaoqu Baijiu, where 2-methylpropanal (Osme values = 3.22; 3.12), 1,1-dimethoxyethane (Osme values $=4.02 ; 3.65)$, hexanal (Osme values $=3.25 ; 3.02)$, and benzeneacetaldehyde (Osme values $=3.65 ; 3.30$ ) had high Osme values above 3.00 
and contributed to malty, fruity, green, and honey odors, respectively. In this study, four ketones were identified. Among them, 2-octanone with soap aroma showed the highest Osme values of 3.22 and 3.20 in $\mathrm{M}$ and T, respectively. All aldehydes and ketones had higher Osme values in the M sample than in the T sample except 1,1,3-triethoxypropane.

The important acids in $\mathrm{M}$ and $\mathrm{T}$ with high Osme values were acetic acid (Osme values $=4.12 ; 4.05)$, 2-methylpropanoic acid (Osme values $=3.25 ; 3.22)$, butanoic acid (Osme values $=3.55 ; 3.95)$, 3-methylbutanoic acid (Osme values $=2.25 ; 2.15)$, pentanoic acid (Osme values $=2.00 ; 1.95)$, and hexanoic acid (Osme values $=2.32 ; 1.75)$. Acids are important components in the quality and taste of alcoholic beverages. Most of the acids in Baijiu are produced by yeast during fermentation, followed by oxidation of alcohol and aldehyde [19]. Acetic acid contributed vinegar aroma while 2-methylpropanoic acid, butanoic acid, 3-methylbutanoic acid, pentanoic acid, and hexanoic acid gave a sweaty odor. Phenols could be important contributors to Baijiu flavor due to their low odor threshold. Among the six phenolic compounds, the Osme values of guaiacol (Osme values $=3.25$; 1.22 ) and 4-ethylguaiacol (Osme values $=0.95 ; 2.15$ ) varied significantly between the $\mathrm{M}$ and $\mathrm{T}$ samples. Five terpenoids were detected in GC-O. Terpenoids not only have a positive aroma contribution to Baijiu flavor but also have healthy physiological activities $[17,20]$. Linalool (Osme values $=3.52 ; 3.85), \beta$-damascenone $($ Osme values $=4.12 ; 3.60)$, and $\beta$ ionone (Osme values $=3.55 ; 2.35$ ) could be important to the aroma of xiaoqu Baijiu because of the high Osme values. Sulfur compounds are very important for Baijiu because of their low thresholds. They are mainly produced by the degradation of sulfur-containing amino acids [21]. Two sulfur compounds, dimethyl trisulfide (Osme values $=4.12 ; 3.50$ ) and 2-thiophenecarboxaldehyde (Osme values $=2.80 ; 2.65)$, were identified in both samples .

\subsection{Quantitation and OAVs of Aroma-Active Compounds in Xiaoqu Baijiu}

To gain a deeper insight into the aroma-active compounds of xiaoqu Baijiu, the 79 odorants detected using GC-O were quantitated by constructing standard curves for the $\mathrm{M}$ and $\mathrm{T}$ samples. The quantitative ion, IS, calibration curve, recovery, and LOQ of each aroma compound are shown in Table 2 and Figure 2. Quantitative results showed that 1,1dimethoxyethane, ethyl acetate, 1-propanol, 2-butanol, 2-methylpropanol, 3-methylbutanol, and acetic acid showed relatively high concentrations (above $100 \mathrm{mg} / \mathrm{L}$ ) in both samples.

(A)

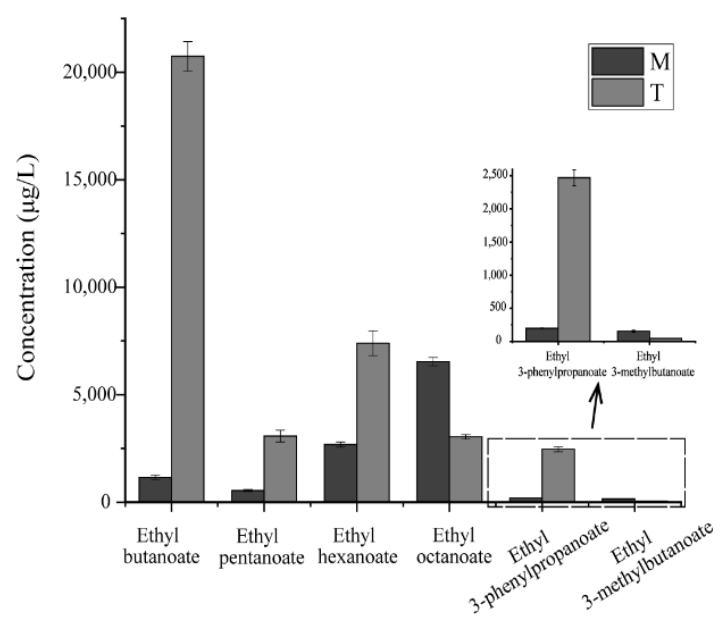

(B)

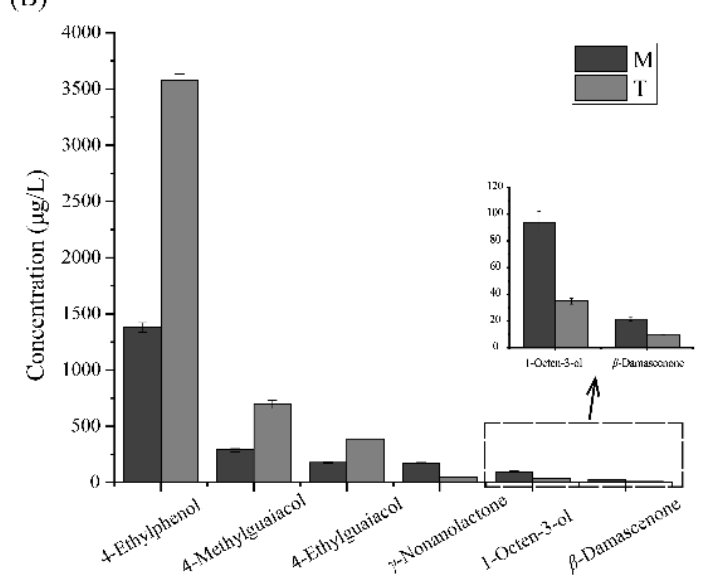

Figure 2. Concentrations of some different aroma-active compounds in $\mathrm{M}$ and $\mathrm{T}$ samples. In the Figure 2, (A) is the concentration difference between ester compounds, and $(\mathbf{B})$ is the concentration difference between others.

It is known that the contribution to Baijiu depends not only on the concentration of an odorant but also its odor threshold. So, for further confirmation of the contribution of aroma-active compounds, their OAVs were calculated. The OAV was obtained from a compound concentration divided by its odor threshold [22]. Herein, the major- 
ity of the threshold values were taken from the literature [23-25]. As shown in Table 3, 42 compounds yielded an $\mathrm{OAV} \geq 1$ in both $\mathrm{M}$ and $\mathrm{T}$ samples, which indicated that these odorants may have a major contribution to the characteristic aroma. The most important aroma compounds (OAV $\geq 100$ ) in $\mathrm{M}$ and/or $\mathrm{T}$ were ethyl octanoate (OAVs $=507.20$; 235.88), 3-methylbutanal (OAVs $=307.74 ; 190.36), \beta$-damascenone $(\mathrm{OAVs}=178.98 ; 78.11)$, dimethyl trisulfide $(\mathrm{OAVs}=161.22 ; 116.14), 1,1$-dimethoxyethane $(\mathrm{OAVs}=140.03 ; 90.33)$, isoamyl acetate $(\mathrm{OAVs}=96.88 ; 132.29)$, ethyl hexanoate $(\mathrm{OAVs}=48.52 ; 133.74)$, ethyl pentanoate $(\mathrm{OAVs}=20.35 ; 114.76)$, and ethyl butanoate $(\mathrm{OAVs}=14.21 ; 254.49)$. These results revealed the important aroma contribution of esters, especially ethyl esters, for xiaoqu Baijiu liquors. Esters, which contribute a strong fruity odor, were also one of the most important aroma groups for other alcoholic beverages, such as wine [26], rice wine [27], and other types of Baijiu [7,15,25,28]. Besides, 3-methylbutanal and acetal ranked at the second and fifth places, respectively, according to their OAV. It is worth noting that the content of $\beta$-damascenone and dimethyl trisulfide was not high but the OAVs ranked at second and third places among all aroma compounds in xiaoqu Baijiu. This is because their thresholds are present at trace amounts in Baijiu [23,25]. $\beta$-Damascenone giving a honey note was previously reported as a key odorant with high OAV in the light aroma type of Baijiu [25,29]. Dimethyl trisulfide, exhibiting cabbage notes, was one of the key aroma compounds in Zhima aroma-type Baijiu and Moutai aroma-type Baijiu [24,30]. The other important odoractive volatiles with OAVs greater than or equal to 10 in $\mathrm{M}$ and / or T were ethyl acetate $(\mathrm{OAVs}=36.49 ; 33.53)$, ethyl 3-methylbutanoate $(\mathrm{OAVs}=22.48 ; 6.56)$, hexanal $(\mathrm{OAVs}=18.21$; 13.76), pentanoic acid $(\mathrm{OAVs}=17.30 ; 19.85)$, 1-octen-3-ol (OAVs $=15.37 ; 5.70), 1$-propanol $(\mathrm{OAVs}=12.53 ; 15.89)$, linalool $(\mathrm{OAVs}=12.39 ; 14.57)$, and 2-methylpropanol $(\mathrm{OAVs}=8.35$; 11.57). Compounds, including ethyl 2-methylpropanoate, ethyl 2-methylbutanoate, guaiacol, 2-butanol, 1-butanol, 3-methylbutanol, ethyl decanoate, ethyl dodecanoate, acetic acid, 2-methylpropanoic acid, butanoic acid, 3-methylbutanoic acid, 4-methylpentanoic acid, hexanoic acid, $\beta$-ionone, ethyl lactate, 4-vinylguaiacol, 4-ethylphenol, and 2-phenethyl acetate, were other aroma contributors.

3.3. Confirmation of the Key Compounds Related to the Aroma Profile Differences between Modern and Traditional Type Xiaoqu Baijiu

As concluded above, the odorant compositions of $\mathrm{M}$ and $\mathrm{T}$ infusions were similar, but the Osme value and OAV of each substance were different. However, these were just the results based on two samples, not enough to represent all the aroma characteristics of modern type xiaoqu Baijiu and traditional type xiaoqu Baijiu. To determine if the differences between $\mathrm{M}$ and $\mathrm{T}$ samples can also be found in an additional 22 different types of xiaoqu Baijiu samples, which were randomly selected from the modern and traditional workshops, the 79 aroma compounds were quantitated. Then, PCA and PLS-DA analyses were conducted based on the concentration of the aroma compounds in the 22 xiaoqu Baijiu samples.

PCA was performed to show a trend of intergroup separation on the scores plot of data obtained in both positive and negative modes. The PCA results showed that five principal components (PCs) with eigenvalues higher than one were obtained from the data sets, whose cumulative variance proportion was $74.5 \%$. PC1 accounted for $46.7 \%$ of the total variance while PC2 explained $7.02 \%$, which explained $55.1 \%$ of the total variance in the data set. As shown in Figure 3A, it was found that 22 xiaoqu Baijiu samples were well separated. PC1 clearly distinguished modern type xiaoqu Baijiu and traditional type xiaoqu Baijiu, traditional type xiaoqu Baijiu was on the positive side of PC1, while modern type $x i a o q u$ Baijiu appeared on the negative side, indicating that there were obvious differences in aroma compounds' features. 
Table 3. Concentrations of aroma compounds, odor thresholds, and odor activity values (OAVs) in Chinese xiaoqu Baijiu.

\begin{tabular}{|c|c|c|c|c|c|c|c|}
\hline \multirow{2}{*}{ No. } & \multirow{2}{*}{ Compound } & \multicolumn{2}{|c|}{ Concentration ( $\mu \mathrm{g} / \mathrm{L})$} & \multirow{2}{*}{$\begin{array}{c}\text { Threshold }^{a} \\
(\mu \mathrm{g} / \mathrm{L})\end{array}$} & \multicolumn{2}{|c|}{ OAV } & \multirow{2}{*}{$\begin{array}{c}\text { Concentration } \\
\text { Ratio }\end{array}$} \\
\hline & & $\mathbf{M}$ & $\mathbf{T}$ & & $\mathbf{M}$ & $\mathbf{T}$ & \\
\hline 16 & Ethyl octanoate & $6542.88 \pm 194.20$ & $3042.84 \pm 107.10$ & $12.9^{b}$ & 507.20 & 235.88 & $2.15^{\mathrm{g}}$ \\
\hline 44 & 3-Methylbutanal & $5077.61 \pm 201.98$ & $3140.96 \pm 99.06$ & $16.5^{b}$ & 307.74 & 190.36 & $1.62^{g}$ \\
\hline 70 & $\beta$-Damascenone & $21.48 \pm 1.22$ & $9.37 \pm 0.23$ & $0.12^{c}$ & 178.98 & 78.11 & $2.29 \mathrm{~g}$ \\
\hline 78 & Dimethyl trisulfide & $70.04 \pm 3.75$ & $53.81 \pm 1.59$ & $0.41^{b}$ & 161.22 & 116.14 & $1.38^{g}$ \\
\hline 43 & 1,1-Dimethoxyethane & $293,200.02 \pm 16,250.65$ & $194,930.69 \pm 12,890.61$ & $2090^{\mathrm{d}}$ & 140.03 & 90.33 & $1.50^{\mathrm{g}}$ \\
\hline 8 & Isoamyl acetate & $9106.74 \pm 855.52$ & $12,434.91 \pm 713.97$ & $94^{\mathrm{b}}$ & 96.88 & 132.29 & $1.37^{\mathrm{h}}$ \\
\hline 12 & Ethyl hexanoate & $2683.21 \pm 121.80$ & $7395.99 \pm 579.91$ & $55.3^{b}$ & 48.52 & 133.74 & $2.76^{\mathrm{h}}$ \\
\hline 1 & Ethyl acetate & $1,189,626.20 \pm 28,686.27$ & $1,093,055.49 \pm 74,210.60$ & $32600^{b}$ & 36.49 & 33.53 & $1.09 \mathrm{~g}$ \\
\hline 7 & $\begin{array}{c}\text { Ethyl } \\
\text { 3-methylbutanoate }\end{array}$ & $155.12 \pm 15.12$ & $45.24 \pm 2.64$ & $6.9^{b}$ & 22.48 & 6.56 & $3.43^{g}$ \\
\hline 9 & Ethyl pentanoate & $545.39 \pm 50.56$ & $3075.6 \pm 276.15$ & $26.8^{b}$ & 20.35 & 114.76 & $5.64^{\mathrm{h}}$ \\
\hline 45 & Hexanal & $464.28 \pm 23.31$ & $350.84 \pm 18.68$ & $25.5^{b}$ & 18.21 & 13.76 & $1.32^{\mathrm{g}}$ \\
\hline 59 & Pentanoic acid & $6731.23 \pm 536.16$ & $7721.93 \pm 122.13$ & $389^{b}$ & 17.30 & 19.85 & $1.15^{\mathrm{h}}$ \\
\hline 36 & 1-Octen-3-ol & $94.04 \pm 8.29$ & $34.87 \pm 2.26$ & $6.12^{c}$ & 15.37 & 5.70 & $2.70^{g}$ \\
\hline 5 & Ethyl butanoate & $1158.52 \pm 98.27$ & $20,740.96 \pm 684.53$ & $81.5^{\mathrm{b}}$ & 14.21 & 254.49 & $17.90^{\mathrm{h}}$ \\
\hline 28 & 1-Propanol & $676,572.64 \pm 62,948.37$ & $858,160.14 \pm 43,057.77$ & $54,000^{\mathrm{b}}$ & 12.53 & 15.89 & $1.27^{\mathrm{h}}$ \\
\hline 69 & Linalool & $162.25 \pm 16.03$ & $190.93 \pm 5.00$ & $13.1^{\mathrm{e}}$ & 12.39 & 14.57 & $1.18^{\mathrm{h}}$ \\
\hline 49 & Benzeneacetaldehyde & $2445.78 \pm 61.59$ & $1404.18 \pm 15.74$ & $262^{d}$ & 9.34 & 5.36 & $1.74^{\mathrm{g}}$ \\
\hline 29 & 2-Methylpropanol & $236,239.43 \pm 18,833.05$ & $327,289.50 \pm 20,690.96$ & $28,300^{c}$ & 8.35 & 11.57 & $1.39^{\mathrm{h}}$ \\
\hline 6 & $\begin{array}{c}\text { Ethyl } \\
\text { 2-methylbutanoate }\end{array}$ & $145.14 \pm 8.87$ & $149.22 \pm 3.49$ & $18^{\mathrm{b}}$ & 8.06 & 8.29 & $1.03^{\mathrm{h}}$ \\
\hline 63 & Guaiacol & $102.93 \pm 6.09$ & $51.96 \pm 2.19$ & $13.4^{\mathrm{b}}$ & 7.68 & 3.88 & $1.98^{g}$ \\
\hline 57 & Butanoic acid & $6842.96 \pm 187.62$ & $10,424.39 \pm 404.47$ & $964^{b}$ & 7.10 & 10.82 & $1.52^{\mathrm{h}}$ \\
\hline 27 & 2-Butanol & $339,921.21 \pm 8741.68$ & $665,096.28 \pm 13,098.88$ & $50,000^{\mathrm{b}}$ & 6.81 & 13.29 & $1.95^{\mathrm{h}}$ \\
\hline 32 & 3-Methylbutanol & $1,165,477.58 \pm 90,842.60$ & $757,555.98 \pm 66,411.82$ & $179,000^{b}$ & 6.51 & 4.23 & $1.54^{\mathrm{g}}$ \\
\hline 30 & 1-Butanol & $17,586.32 \pm 1058.58$ & $27,280.65 \pm 1578.84$ & $2730^{\mathrm{b}}$ & 6.44 & 9.99 & $1.55^{\mathrm{h}}$ \\
\hline 25 & Ethyl dodecanoate & $2507.65 \pm 244.90$ & $1471.96 \pm 106.06$ & $400^{\mathrm{f}}$ & 6.27 & 3.68 & $1.70^{g}$ \\
\hline 58 & 3-Methylbutanoic acid & $6300.25 \pm 75.10$ & $5426.10 \pm 351.74$ & $1050^{b}$ & 6.00 & 5.17 & $1.16^{\mathrm{g}}$ \\
\hline 19 & Ethyl decanoate & $5867.76 \pm 366.2$ & $5123.68 \pm 182.18$ & $1120^{b}$ & 5.24 & 4.57 & $1.15^{\mathrm{g}}$ \\
\hline 3 & $\begin{array}{c}\text { Ethyl } \\
\text { 2-methylpropanoate }\end{array}$ & $240.10 \pm 15.57$ & $262.45 \pm 15.12$ & $57.5^{b}$ & 4.18 & 4.56 & $1.09^{\mathrm{h}}$ \\
\hline 73 & $\beta$-Ionone & $5.28 \pm 0.20$ & $3.86 \pm 0.31$ & $1.3^{\mathrm{d}}$ & 4.06 & 2.97 & $1.37^{g}$ \\
\hline 14 & Ethyl lactate & $510,767.23 \pm 970.08$ & $484,485.04 \pm 387.49$ & $128,000^{b}$ & 3.99 & 3.79 & $1.05^{\mathrm{g}}$ \\
\hline 60 & 4-Methylpentanoic acid & $567.06 \pm 16.66$ & $835.88 \pm 30.11$ & $144^{\mathrm{c}}$ & 3.94 & 5.80 & $1.47^{\mathrm{h}}$ \\
\hline 67 & 4-Vinylguaiacol & $691.08 \pm 24.02$ & $804.60 \pm 16.15$ & $209^{b}$ & 3.30 & 3.84 & $1.16^{\mathrm{h}}$ \\
\hline 56 & $\begin{array}{l}\text { 2-Methylpropanoic } \\
\text { acid }\end{array}$ & $5131.08 \pm 428.69$ & $4225.58 \pm 286.11$ & $1580^{\mathrm{d}}$ & 3.25 & 2.68 & $1.21^{\mathrm{g}}$ \\
\hline 54 & Acetic acid & $557,164.75 \pm 54,070.93$ & $426,675.42 \pm 22,473.95$ & $200,000^{b}$ & 2.79 & 2.14 & $1.31^{\mathrm{g}}$ \\
\hline 68 & 4-Ethyl phenol & $1379.60 \pm 42.75$ & $3573.64 \pm 58.21$ & $618^{\mathrm{b}}$ & 2.24 & 5.78 & $2.59^{\mathrm{h}}$ \\
\hline 23 & 2-Phenethyl acetate & $1870.82 \pm 198.57$ & $1970.58 \pm 188.82$ & $909^{b}$ & 2.06 & 2.17 & $1.05^{\mathrm{h}}$ \\
\hline 61 & Hexanoic acid & $5046.77 \pm 331.75$ & $3916.83 \pm 236.21$ & $2520^{b}$ & 2.00 & 1.55 & $1.29 \mathrm{~g}$ \\
\hline 46 & 1,1,3-Triethoxypropane & $7268.03 \pm 576.60$ & $12,143.02 \pm 793.82$ & $3700^{b}$ & 1.97 & 3.28 & $1.67^{\mathrm{h}}$ \\
\hline 77 & $\gamma$-Nonanolactone & $172.01 \pm 6.48$ & $49.76 \pm 1.13$ & $90.7^{\mathrm{b}}$ & 1.90 & 0.55 & $3.46^{\mathrm{g}}$ \\
\hline 55 & Propanoic acid & $31,045.80 \pm 2132.72$ & $37,776.75 \pm 1305.67$ & $18,200^{b}$ & 1.71 & 2.08 & $1.22^{\mathrm{h}}$ \\
\hline 24 & $\begin{array}{c}\text { Ethyl } \\
\text { 3-phenylpropanoate }\end{array}$ & $199.54 \pm 6.45$ & $2470.88 \pm 120.61$ & $130^{b}$ & 1.54 & 19.01 & $12.34^{\mathrm{h}}$ \\
\hline 65 & 4-Ethylguaiacol & $178.16 \pm 5.15$ & $387.86 \pm 2.40$ & $123^{\mathrm{b}}$ & 1.44 & 3.16 & $2.18^{\mathrm{h}}$ \\
\hline 41 & $\beta$-Phenethyl alcohol & $40,101.93 \pm 2462.57$ & $23,215.7 \pm 1252.70$ & $28,900^{b}$ & 1.39 & 0.80 & $1.73^{\mathrm{g}}$ \\
\hline 42 & 2-Methyl propanal & $1708.38 \pm 140.81$ & $1525.56 \pm 149.01$ & $1300^{\mathrm{b}}$ & 1.31 & 1.14 & $1.12^{\mathrm{g}}$ \\
\hline 64 & 4-Methylguaiacol & $290.22 \pm 18.08$ & $694.76 \pm 36.65$ & $315^{b}$ & 0.92 & 2.21 & $2.39^{\mathrm{h}}$ \\
\hline 62 & Octanoic acid & $2374.17 \pm 36.35$ & $1423.26 \pm 90.68$ & $2700^{b}$ & 0.88 & 0.53 & $1.67^{g}$ \\
\hline 10 & Pentyl acetate & $142.56 \pm 7.20$ & $288.16 \pm 7.20$ & $180^{\mathrm{d}}$ & 0.80 & 1.60 & $2.02^{\mathrm{h}}$ \\
\hline 2 & Ethyl propionate & $10,868.83 \pm 544.43$ & $12,827.82 \pm 1141.29$ & $19,000^{b}$ & 0.57 & 0.68 & $1.18^{\mathrm{h}}$ \\
\hline 47 & Decanal & $40.61 \pm 3.19$ & $60.12 \pm 4.03$ & $70.8^{\mathrm{d}}$ & 0.57 & 0.85 & $1.48^{\mathrm{h}}$ \\
\hline 34 & 1-Hexanol & $2740.72 \pm 90.84$ & $3820.35 \pm 140.62$ & $5370^{\mathrm{d}}$ & 0.50 & 0.70 & $1.39^{\mathrm{h}}$ \\
\hline 33 & 2-Heptanol & $685.65 \pm 16.11$ & $85.65 \pm 5.60$ & $1430^{d}$ & 0.48 & 0.06 & $8.01^{\mathrm{g}}$ \\
\hline 66 & 4-Methyl phenol & $55.82 \pm 3.26$ & $39.10 \pm 6.93$ & $167^{\mathrm{b}}$ & 0.34 & 0.24 & $1.43^{\mathrm{g}}$ \\
\hline 51 & 2-Octanone & $64.45 \pm 5.99$ & $58.59 \pm 5.80$ & $250^{c}$ & 0.26 & 0.23 & $1.10^{\mathrm{g}}$ \\
\hline 39 & 1-Nonanol & $187.91 \pm 11.48$ & $136.68 \pm 8.94$ & $806^{d}$ & 0.23 & 0.17 & $1.37^{g}$ \\
\hline 35 & 3-Octanol & $86.50 \pm 5.15$ & $106.75 \pm 7.84$ & $393^{c}$ & 0.22 & 0.27 & $1.23^{g}$ \\
\hline 52 & Acetophenone & $50.55 \pm 2.91$ & $6.82 \pm 0.41$ & $256^{\mathrm{d}}$ & 0.20 & 0.03 & $7.41^{\mathrm{g}}$ \\
\hline 75 & Furfural & $6568.22 \pm 651.46$ & $2581.56 \pm 113.18$ & $44,000^{\mathrm{b}}$ & 0.15 & 0.06 & $2.54^{g}$ \\
\hline 22 & Ethyl phenylacetate & $44.49 \pm 2.99$ & $35.24 \pm 2.59$ & $407^{\mathrm{b}}$ & 0.11 & 0.09 & $1.26^{\mathrm{g}}$ \\
\hline 17 & Ethyl nonanoate & $326.85 \pm 32.41$ & $173.76 \pm 15.58$ & $3200^{b}$ & 0.10 & 0.05 & $1.88^{\mathrm{g}}$ \\
\hline
\end{tabular}


Table 3. Cont

\begin{tabular}{|c|c|c|c|c|c|c|c|}
\hline \multirow{2}{*}{ No. } & \multirow{2}{*}{ Compound } & \multicolumn{2}{|c|}{ Concentration $(\mu \mathrm{g} / \mathrm{L})$} & \multirow{2}{*}{$\begin{array}{c}\text { Threshold }^{a} \\
(\mu \mathrm{g} / \mathrm{L})\end{array}$} & \multicolumn{2}{|c|}{ OAV } & \multirow{2}{*}{$\begin{array}{c}\text { Concentration } \\
\text { Ratio }\end{array}$} \\
\hline & & $\mathbf{M}$ & $\mathbf{T}$ & & $\mathbf{M}$ & $\mathbf{T}$ & \\
\hline 72 & Geranylacetone & $23.83 \pm 1.82$ & $18.81 \pm 0.29$ & $267^{\mathrm{f}}$ & 0.09 & 0.07 & $1.27^{\mathrm{g}}$ \\
\hline 48 & Benzaldehyde & $317.00 \pm 23.26$ & $151.23 \pm 1.24$ & $4200^{b}$ & 0.08 & 0.04 & $2.10^{g}$ \\
\hline 76 & 2-Furan methanol & $4178.60 \pm 104.80$ & $2012.85 \pm 46.29$ & $54,700^{\mathrm{c}}$ & 0.06 & 0.04 & $1.56^{\mathrm{g}}$ \\
\hline 20 & Diethyl succinate & $18,214.34 \pm 1109.16$ & $11,493.28 \pm 865.21$ & $353,000^{b}$ & 0.05 & 0.03 & $1.58^{\mathrm{g}}$ \\
\hline 71 & Geraniol & $5.78 \pm 0.19$ & $3.01 \pm 0.29$ & $120^{\mathrm{f}}$ & 0.05 & 0.03 & $1.92^{\mathrm{g}}$ \\
\hline 11 & $\begin{array}{l}\text { 3-Methylbutyl } \\
\text { butanoate }\end{array}$ & $35.15 \pm 3.45$ & $431.55 \pm 38.24$ & $915^{\mathrm{d}}$ & 0.04 & 0.47 & $12.28^{h}$ \\
\hline 38 & 1-Octanol & $33.00 \pm 1.37$ & $13.56 \pm 0.86$ & $1100^{c}$ & 0.03 & 0.01 & $2.43^{g}$ \\
\hline 31 & 1-Pentanol & $1052.35 \pm 103.83$ & $1639.97 \pm 135.46$ & $37,400^{b}$ & 0.03 & 0.04 & $1.56^{\mathrm{h}}$ \\
\hline 40 & Benzyl alcohol & $1024.33 \pm 40.75$ & $2302.44 \pm 81.79$ & $40,900^{b}$ & 0.03 & 0.06 & $2.25^{\mathrm{h}}$ \\
\hline 21 & Ethyl benzoate & $22.15 \pm 2.02$ & $3.78 \pm 0.23$ & $1400^{b}$ & 0.02 & $<0.01$ & $5.86^{\mathrm{g}}$ \\
\hline 4 & Isobutyl acetate & $16.38 \pm 1.39$ & $49.14 \pm 4.58$ & $922^{b}$ & 0.02 & 0.05 & $3.00^{\mathrm{h}}$ \\
\hline 15 & Ethyl heptanoate & $195.83 \pm 18.23$ & $204.63 \pm 13.15$ & $13,200^{b}$ & 0.01 & 0.02 & $1.04^{\mathrm{h}}$ \\
\hline 37 & 1-Heptanol & $174.40 \pm 2.79$ & $106.98 \pm 6.02$ & $26,600^{d}$ & 0.01 & $<0.01$ & $1.63^{g}$ \\
\hline 13 & Propyl hexanoate & $34.25 \pm 2.54$ & $129.31 \pm 8.21$ & $13,000^{\mathrm{d}}$ & $<0.01$ & 0.01 & $3.78^{\mathrm{h}}$ \\
\hline 18 & Hexyl hexanoate & $3.96 \pm 0.25$ & $15.73 \pm 1.16$ & $1890^{\mathrm{d}}$ & $<0.01$ & 0.01 & $3.97^{\mathrm{h}}$ \\
\hline 26 & Ethyl tetradecanoate & $835.26 \pm 44.31$ & $454.07 \pm 22.18$ & $494,000^{b}$ & $<0.01$ & $<0.01$ & $1.84^{\mathrm{g}}$ \\
\hline 50 & 2-Pentanone & $775.20 \pm 60.42$ & $346.25 \pm 10.92$ & - & & & $2.24^{g}$ \\
\hline 74 & 2-Pentylfuran & $751.32 \pm 57.57$ & $466.86 \pm 29.76$ & - & & & $1.61^{\mathrm{g}}$ \\
\hline 79 & Thiophenecarboxaldehyde & $858.04 \pm 47.96$ & $556.41 \pm 16.91$ & - & & & $1.54^{\mathrm{g}}$ \\
\hline 53 & 2-Pentadecanone & $3.84 \pm 0.27$ & $3.40 \pm 0.09$ & - & & & $1.13^{\mathrm{g}}$ \\
\hline
\end{tabular}

a Odor thresholds were taken from reference. ${ }^{b}$ Odor thresholds taken from reference [22,23]. ${ }^{c}$ Odor thresholds taken from reference [24]. ${ }^{\mathrm{d}}$ Odor thresholds taken from reference [25]. ${ }^{\mathrm{e}}$ Odor thresholds taken from reference [31]. ${ }^{\mathrm{f}}$ Odor thresholds taken from reference [13]. $\mathrm{g}$ The concentration ratio of $\mathrm{M}$ to $\mathrm{T}$. $\mathrm{h}$ The concentration ratio of $\mathrm{T}$ to M.Identified odorants with high OAVs can also be used as indicators to assess aroma differences objectively in the two samples. Among the compounds with OAVs $\geq 1$, we found that the concentrations of ethyl octanoate, $\beta$-damascenone, 3-methylbutanal, ethyl 3-methylbutanoate, benzeneacetaldehyde, 1-octen-3-ol, guaiacol, 1,1-dimethoxyethane, 3-methylbutanol, ethyl dodecanoate, $\gamma$-nonanolactone, and $\beta$-phenethyl alcohol, were $1.50-17.9$ times higher in the $\mathrm{M}$ than in the T sample. In contrast, the concentrations of ethyl butanoate, ethyl pentanoate, ethyl hexanoate, 1-butanol, 2-butanol, butanoic acid, 1,1,3-triethoxypropane, 4-ethylphenol, 4-methylguaiacol, ethyl 3-phenylpropanoate, and 4-ethylguaiacol were 1.52-24.77 times higher in the T sample than in the M sample. The 23 aroma compounds might be potential compounds responsible for the differences in the aroma profile between $\mathrm{M}$ and $\mathrm{T}$ samples. Figure 2 shows the concentration of the 12 aroma-active compounds (concentration ratio $\geq 2$ ) in the $\mathrm{M}$ and T samples.

(A)

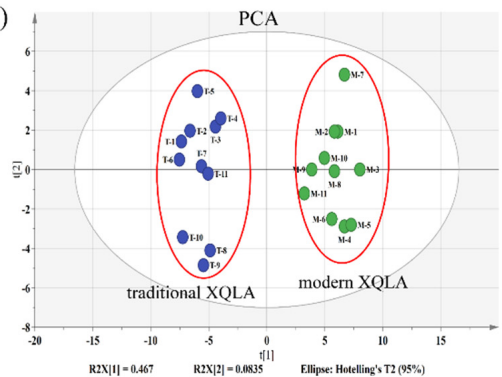

(C)

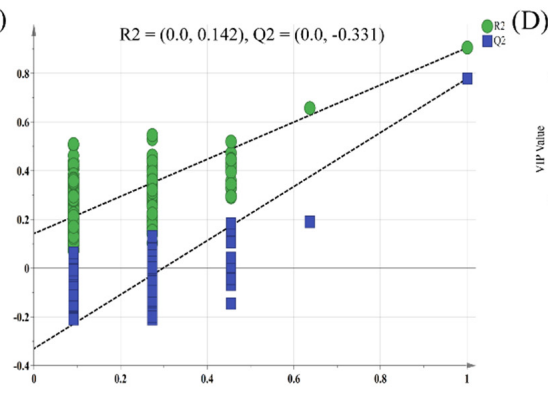

B)

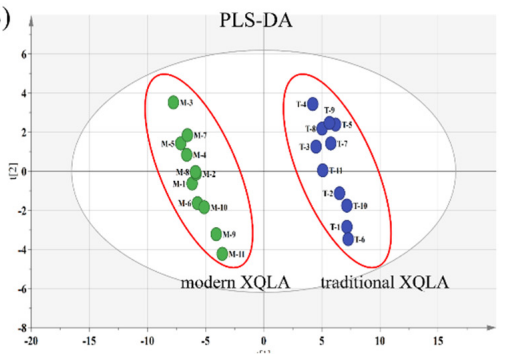

(D)

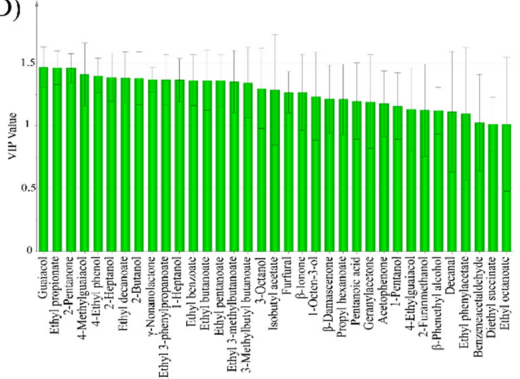

Figure 3. (A) PCA (Principal component analysis) scores scatter plot for modern xiaoqu Baijiu and traditional xiaoqu Baijiu samples (PC1 46.7\%, PC2 8.35\%). (B) PLS-DA (partial least squares discriminant analysis) scores scatter plot for different samples (R2Y $=0.993$ and Q2 $=0.978)$. $(\mathbf{C})$ The validate model of the PLS-DA model. (D) The variable importance for projection (VIP) plot (VIP > 1). 
To maximize separation and identification of the aroma compound markers between the two types of xiaoqu Baijiu, a PLS-DA model was created for the 22 xiaoqu Baijiu samples based on the concentration data set. This type of supervised pattern recognition method provides a deeper analysis of the main matrix characteristics of samples and differs from PCA by the addition of grouping variables that indicate the category of samples. As shown in Figure 3B, the two types of xiaoqu Baijiu samples were well distinguished by a dependable PLS-DA model. The explained variation $\left(R^{2} Y\right)$ and the predictive capability $\left(Q^{2} Y\right)$ for the model were 0.993 and 0.978 , respectively, which indicated that the model was excellent because $Q^{2} Y$ was close to 1 . In addition, permutation tests $(n=200)$ were performed to evaluate whether the discriminant models were overfitting the data [32]. The permutation tests randomly rearranged the experiments by changing the sort order of the classification variables $(\mathrm{Y})$ and randomly assigned $\mathrm{Q}^{2} \mathrm{Y}$ up to 200 times. The low intercepts $\left(\mathrm{R}^{2}=0.142, \mathrm{Q}^{2}=-0.331\right)$ were an indication of the validity of the original model. The $\mathrm{R}^{2}$ and $\mathrm{Q}^{2}$ values of the permutation test revealed that the initial model outperformed the randomly permuted models (Figure 3C). Variables with absolute variable importance for projection (VIP) values greater than 1 were selected as potential markers explaining the differences. As shown in Figure 3D, a total of 35 compounds had a VIP value greater than 1, and 20 out of the 35 compounds were important contributors to the aroma profile of xiaoqu Baijiu, which indicated that they were highly relevant to the difference between modern type xiaoqu Baijiu and traditional type xiaoqu Baijiu samples. These 20 substances cover most of the different substances obtained by comparing the concentrations of the $\mathrm{M}$ and $\mathrm{T}$ samples. This showed that the multivariate statistical analyses of 22 samples verified the results of Section 3.2.

To compare further the content of each substance in the two types of xiaoqu Baijiu, the concentrations of 20 potential aroma-active compounds in the samples from both the modern type xiaoqu Baijiu and traditional type xiaoqu Baijiu were used to prepare HCA analysis, and the color (from light to dark) indicated the concentration change from low to high. As shown in Figure 4, the HCA clearly classified the aroma markers into two clusters. Cluster A consists of the aroma-active compounds with significantly higher concentrations in traditional type $x i a o q u$ Baijiu than in modern type xiaoqu Baijiu, which included five ethyl esters (ethyl 3-phenylpropanoate, ethyl propanoate, ethyl butanoate, ethyl pentanoate, and ethyl phenylacetate), three phenols (4-ethylphenol, 4-ethylguaiacol, and 4-methylguaiacol), pentanoic acid, $\beta$-ionone, and 2-butanol.

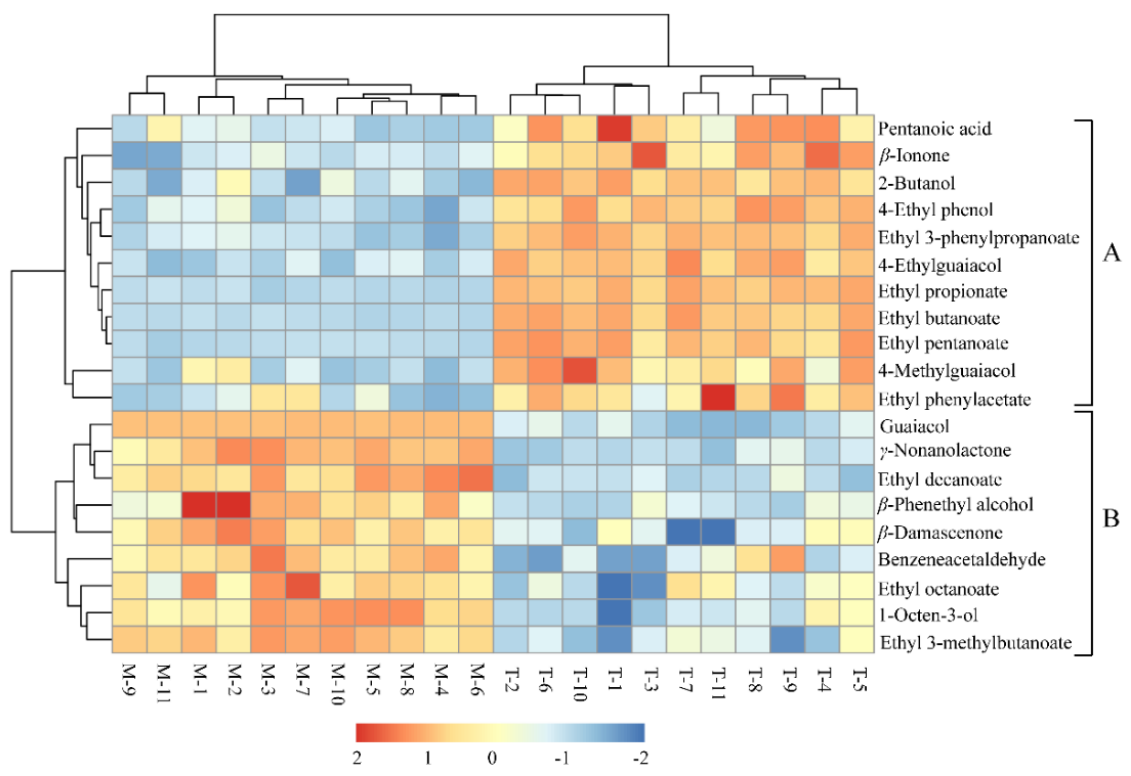

Figure 4. Heat map of the key aroma compounds maker between modern type xiaoqu Baijiu and traditional type xiaoqu Baijiu. 
The ethyl esters in Baijiu were mainly produced in the fermentation or aging stages through the esterification of acids and alcohols $[7,33]$. This study found that the contents of propanoic acid, butanoic acid, and pentanoic acid in traditional type xiaoqu Baijiu were higher than those in modern type xiaoqu Baijiu, so the organic acids had a significant influence on the yield of aroma esters. Phenols, as an important trace component in Baijiu, play an important role in the aroma, taste, and stability of Baijiu [34]. It was reported that phenols come from the thermal degradation of lignin in brewing raw materials or are produced by amino acids and ferulic acid under the action of microorganisms $[35,36]$. The brewing materials of modern type xiaoqu Baijiu and traditional type xiaoqu Baijiu are the same, therefore, it may be that the different types and abundance of microorganisms caused the higher content of phenols in traditional type xiaoqu Baijiu. $\beta$-Ionone with a floral aroma is also a very important aroma compound and bioactive substance in Baijiu [37]. Besides, 2-butanol is one of the higher alcohols in Baijiu, it is mostly synthesized by microorganisms (mainly Saccharomyces cerevisiae and bacteria) during fermentation via catabolic and anabolic pathways [38,39]. The fermentation temperature of traditional type xiaoqu Baijiu is highly susceptible to climatic conditions, but the fermentation temperature of modern type xiaoqu Baijiu is controlled at a suitable temperature, which could effectively inhibit the production of higher alcohols [13]. Cluster B consists of the aroma-active compounds with significantly higher concentrations in modern type xiaoqu Baijiu than in traditional type xiaoqu Baijiu, which included three esters (ethyl 3-methylbutanoate, ethyl octanoate, and ethyl decanoate), guaiacol, $\gamma$-nonanolactone, $\beta$-phenethyl alcohol, $\beta$ damascenone, benzeneacetaldehyde, and 1-octen-3-ol. Not surprisingly, the acids content corresponding to these three esters in modern type xiaoqu Baijiu was also higher than in traditional type xiaoqu Baijiu. $\beta$-Phenethyl alcohol with a rose-like aroma is a very important aroma compound in Baijiu. The processes by which the key odorant is formed have been well-established and found to be influenced by various yeast species present during the fermentation of liquor from cereal and legume materials [40]. $\beta$-Damascenone, presenting honey and floral aromas, was previously reported as an important odorant in whiskey, rum, brandy, and Baijiu [25,28,41-43]. Luigi and Peter [43] assumed that cereals could contain the precursor (3-hydroxy-7,8-dihydro- $\beta$-ionol) leading to the generation of $\beta$-damascenone during fermentation and/or distillation.

\section{Conclusions}

In summary, a total of 79 aroma compounds were identified using GC-O and GC-MS in both $\mathrm{M}$ and $\mathrm{T}$ samples. Among them, 42 aroma compounds were further recognized as the important aroma-active compounds in both $\mathrm{M}$ and $\mathrm{T}$ owing to their relatively high OAVs. Twenty-three aroma-active compounds might be the potential compounds responsible for the differences in the aroma profile between $\mathrm{M}$ and $\mathrm{T}$ samples because of a concentration ratio higher than 1.5. Moreover, to determine if the 23 aroma-active compounds can represent the difference in aroma compounds' profile between traditional type xiaoqu Baijiu and modern type xiaoqu Baijiu, multivariate analyses of the 22 xiaoqu Baijiu samples made by different brewing processes were applied, including PCA, PLS-DA, and HCA. Twenty odor-active compounds were confirmed as potential flavor markers for the differentiation of modern type xiaoqu Baijiu and traditional type xiaoqu Baijiu. The results in this study will help improve the flavor quality and processing technology of xiaoqu Baijiu.

Author Contributions: Conceptualization, S.C. (Shuang Chen); Data curation, J.T. and S.F.; Methodology, S.C. (Shuang Chen), J.T. and S.F.; Resources, Q.Y. and Y.X.; Supervision, Q.Y. and Y.X.; Writing-original draft, S.C. (Shuang Chen), J.T. and S.F.; Writing—review \& editing, J.Z., S.C. (Shenxi Chen), Y.L., Q.Y. and Y.X. All authors have read and agreed to the published version of the manuscript.

Funding: This research was funded by the National Natural Science Foundation of China (NO. 32172331), Hubei Provincial Key Research and Development Program (NO. 2020BBA050), and Project funded by China Postdoctoral Science Foundation (NO. 2018M631971). 
Institutional Review Board Statement: Not applicable.

Informed Consent Statement: Not applicable.

Data Availability Statement: The data that support the findings of this study are available from the corresponding author upon request.

Conflicts of Interest: The authors declare no conflict of interest.

\section{References}

1. Anal, A. Quality ingredients and safety concerns for traditional fermented foods and beverages from Asia: A review. Fermentation 2019, 5, 8. [CrossRef]

2. Chaves-López, C.; Serio, A.; Grande-Tovar, C.D.; Cuervo-Mulet, R.; Delgado-Ospina, J.; Paparella, A. Traditional fermented foods and beverages from a microbiological and nutritional perspective: The colombian heritage. Compre. Rev. Food Sci. Saf. 2014, 13, 1031-1048. [CrossRef]

3. Marco, M.L.; Heeney, D.; Binda, S.; Cifelli, C.J.; Cotter, P.D.; Foligne, B.; Ganzle, M.; Kort, R.; Pasin, G.; Pihlanto, A.; et al. Health benefits of fermented foods: Microbiota and beyond. Curr. Opin. Biotech. 2017, 44, 94-102. [CrossRef] [PubMed]

4. Zhu, Y.; Tramper, J. Koji-where East meets West in fermentation. Biotechnol. Adv. 2013, 31, 1448-1457. [CrossRef]

5. Settanni, L.; Moschetti, G. Non-starter lactic acid bacteria used to improve cheese quality and provide health benefits. Food Microbiol. 2010, 27, 691-697. [CrossRef]

6. Jin, G.; Zhu, Y.; Xu, Y. Mystery behind Chinese liquor fermentation. Trends Food Sci. Technol. 2017, 63, 18-28. [CrossRef]

7. Zhao, D.; Shi, D.; Sun, J.; Li, A.; Sun, B.; Zhao, M.; Chen, F.; Sun, X.; Li, H.; Huang, M.; et al. Characterization of key aroma compounds in Gujinggong Chinese Baijiu by gas chromatography-olfactometry, quantitative measurements, and sensory evaluation. Food Res. Int. 2018, 105, 616-627. [CrossRef] [PubMed]

8. National Bureau of Statistics of China. Output of Main Industrial Products of Baijiu. Available online: https://data.stats.gov.cn/ easyquery.htm?cn=A01\&zb=A020909\&sj=202012 (accessed on 4 September 2021).

9. Li, Y.; Qi, S.; Qiu, L.; Meng, F. Research progress in and discussion on mechanized production of Baijiu. Liquor Making Sci. Technol. 2016, 10, 82-84. [CrossRef]

10. Ye, H.; Wang, J.; Shi, J.; Du, J.; Zhou, Y.; Huang, M.; Sun, B. Automatic and Intelligent Technologies of Solid-State Fermentation Process of Baijiu Production: Applications, Challenges, and Prospects. Foods. 2021, 10, 680. [CrossRef] [PubMed]

11. Wang, X.; Du, H.; Zhang, Y.; Xu, Y. Environmental microbiota drives microbial succession and metabolic profiles during Chinese liquor fermentation. Appl. Environ. Microbiol. 2018, 84, e02369-17. [CrossRef]

12. Gong, S.; Fan, W.; Yan, X. Comparison of volatile and non-volatile compounds between traditional and mechanical raw Baijiu of roasted-sesame-like aroma type Baijiu(Chinese liquor). Food Ferment. Ind. 2018, 8, 239-245. [CrossRef]

13. Sun, X.; Du, J.; Huang, P.; Zhang, F.; Liu, Y. Analysis of sensory characterization and flavor composition in Xiaoqu liquor made by modern technology and traditional technology. Food Sci. 2021, 42, 282-290. [CrossRef]

14. Polá, K.P.; Herszage, J.; Ebeler, S.E. Wine flavor: Chemistry in a glass. Chem. Soc. Rev. 2008, 37, 2478-2489. [CrossRef]

15. Li, H.; Qin, D.; Wu, Z.; Sun, B.; Sun, X.; Huang, M.; Sun, J.; Zheng, F. Characterization of key aroma compounds in Chinese Guojing sesame-flavor Baijiu by means of molecular sensory science. Food Chem. 2019, 284, 100-107. [CrossRef] [PubMed]

16. Zhang, X.; Wang, C.; Wang, L.; Chen, S.; Xu, Y. Optimization and validation of a head space solid-phase microextraction-arrow gas chromatography-mass spectrometry method using central composite design for determination of aroma compounds in Chinese liquor (Baijiu). J. Chromatogr. A 2020, 1610, 460584. [CrossRef] [PubMed]

17. Qian, Y.; An, Y.; Chen, S.; Qian, M.C. Characterization of Qingke liquor aroma from Tibet. J. Agric. Food Chem. 2019, 67, 13870-13881. [CrossRef]

18. Fan, W.; Qian, M.C. Characterization of aroma compounds of Chinese "Wuliangye" and "Jiannanchun" liquors by aroma extract dilution analysis. J. Agric. Food Chem. 2006, 54, 2659-2704. [CrossRef]

19. Fan, W.; Qian, M.C. Identification of aroma compounds in Chinese 'Yanghe Daqu' liquor by normal phase chromatography fractionation followed by gas chromatography-olfactometry. Flavour Fragr. J. 2006, 21, 333-342. [CrossRef]

20. Li, Q.; Wang, G.; Huang, F.; Banda, M.; Reed, E. Antineoplastic effect of beta-elemene on prostate cancer cells and other types of solid tumour cells. J. Pharm. Pharmacol. 2010, 62, 1018-1027. [CrossRef] [PubMed]

21. Fang, Y.; Qian, M.C. Sensitive quantification of sulfur compounds in wine by headspace solid-phase microextraction technique. J. Chromatogr. A 2005, 1080, 177-185. [CrossRef]

22. Schieberle, P. Chapter 17-New developments in methods for analysis of volatile flavor compounds and their precursors. Charact. Food 1995, 65, 403-431.

23. Fan, W.; Xu, Y. Determination of odor thresholds of volatile aroma compounds in Baijiu by a forced-choice ascending concentration series method of limits. Liquor Mak. 2011, 38, 80-84. [CrossRef]

24. Wang, L.; Fan, S.; Yan, Y.; Yang, L.; Chen, S.; Xu, Y. Characterization of potent odorants causing a pickle-like off-odor in Moutai-aroma type Baijiu by comparative aroma extract dilution analysis, quantitative measurements, aroma addition, and omission studies. J. Agric. Food Chem. 2020, 68, 1666-1677. [CrossRef] 
25. Gao, W.; Fan, W.; Xu, Y. Characterization of the key odorants in light aroma type chinese liquor by gas chromatographyolfactometry, quantitative measurements, aroma recombination, and omission studies. J. Agric. Food Chem. 2014, 62, 5796-5804. [CrossRef] [PubMed]

26. Ma, Y.; Tang, K.; Xu, Y.; Li, J. Characterization of the key aroma compounds in Chinese Vidal icewine by gas chromatographyolfactometry, quantitative measurements, aroma recombination, and omission tests. J. Agric. Food Chem. 2017, 65, 394-401. [CrossRef]

27. Chen, S.; Wang, C.; Qian, M.C.; Li, Z.; Xu, Y. Characterization of the key aroma compounds in aged Chinese rice wine by comparative aroma extract dilution analysis, quantitative measurements, aroma recombination, and omission studies. J. Agric. Food Chem. 2019, 67, 4876-4884. [CrossRef] [PubMed]

28. Fan, H.; Fan, W.; Xu, Y. Characterization of key odorants in Chinese Chixiang aroma-type liquor by gas chromatographyolfactometry, quantitative measurements, aroma recombination, and omission studies. J. Agric. Food Chem. 2015, 63, 3660-3668. [CrossRef]

29. Niu, Y.; Yao, Z.; Xiao, Q.; Xiao, Z.; Ma, N.; Zhu, J. Characterization of the key aroma compounds in different light aroma type Chinese liquors by GC-olfactometry, GC-FPD, quantitative measurements, and aroma recombination. Food Chem. 2017, 233, 204-215. [CrossRef] [PubMed]

30. Zheng, Y.; Sun, B.; Zhao, M.; Zheng, F.; Huang, M.; Sun, J.; Sun, X.; Li, H. Characterization of the key odorants in Chinese Zhima aroma-type Baijiu by gas chromatography-olfactometry, quantitative measurements, aroma recombination, and omission studies. J. Agric. Food Chem. 2016, 64, 5367-5374. [CrossRef]

31. Wang, L.; Hu, G.; Lei, L.; Lin, L.; Wang, D.; Wu, J. Identification and aroma impact of volatile terpenes in Moutai liquor. Int. J. Food Prop. 2015, 16, 1335-1352. [CrossRef]

32. Belmonte-Sanchez, J.R.; Romero-Gonzalez, R.; Arrebola, F.J.; Vidal, J.L.M.; Garrido Frenich, A. An innovative metabolomic approach for golden rum classification combining ultrahigh-performance liquid chromatography-orbitrap mass spectrometry and chemometric strategies. J. Agric. Food Chem. 2019, 67, 1302-1311. [CrossRef] [PubMed]

33. González-Rompinelli, E.M.; Rodríguez-Bencomo, J.J.; García-Ruiz, A.; Sánchez-Patán, F.; Martín-Álvarez, P.J.; Bartolomé, B.; Moreno-Arribas, M.V. A winery-scale trial of the use of antimicrobial plant phenolic extracts as preservatives during wine ageing in barrels. Food Control 2013, 33, 440-447. [CrossRef]

34. Shi, D.; Wang, S.; Zhao, D.; Sun, J.; Li, A.; Sun, X.; Li, H.; Sun, B. Determination of 6 phenols in 103 kinds of Chinese baijiu by GC-MS/SIM. J. Chin. Ins. Food Sci. Technol. 2019, 19, 235-248. [CrossRef]

35. Jiang, Y.; Zhao, D.; Sun, J.; Luo, X.; Li, H.; Sun, X.; Zheng, F. Analysis of antioxidant effect of two tripeptides isolated from fermented grains (Jiupei) and the antioxidative interaction with 4-methylguaiacol, 4-ethylguaiacol, and vanillin. Food Sci. Nutr. 2019, 7, 2391-2403. [CrossRef] [PubMed]

36. Zheng, J.; Liang, R.; Wu, C.; Zhou, R.; Liao, X. Discrimination of different kinds of Luzhou-flavor raw liquors based on their volatile features. Food Res. Int. 2014, 56, 77-84. [CrossRef]

37. Li, Q.; Fan, H.; Shi, B.; Li, W.; Zhang, M.; Xu, H.; Chen, X. Healthcare value and bioactive aubstances of Dongjiu (III). Liquor Making Sci. Technol. 2020, 8, 108-117. [CrossRef]

38. Willaert, R.; Nedovic, V.A. Primary beer fermentation by immobilised yeast-a review on flavour formation and control strategies. J. Chem. Technol. Biot. 2006, 81, 1353-1367. [CrossRef]

39. Jiang, J.; Liu, Y.; Li, H.; Yang, Q.; Wu, Q.; Chen, S.; Tang, J.; Xu, Y. Modeling and Regulation of Higher Alcohol Production through the Combined Effects of the C/N Ratio and Microbial Interaction. J. Agric. Food Chem. 2019, 67, 10694-10701. [CrossRef]

40. Carmona, M.; Zamarro, M.T.; Blazquez, B.; Durante-Rodriguez, G.; Juarez, J.F.; Valderrama, J.A.; Barragan, M.J.L.; Garcia, J.L.; Diaz, E. Anaerobic catabolism of aromatic compounds: A genetic and genomic view. Microbiol. Mol. Biol. Rev. 2009, 73, 71-133. [CrossRef]

41. Pino, J.A.; Tolle, S.; Gok, R.; Winterhalter, P. Characterisation of odour-active compounds in aged rum. Food Chem. 2012, 132, 1436-1441. [CrossRef]

42. Willner, B.; Granvogl, M.; Schieberle, P. Characterization of the key aroma compounds in Bartlett pear brandies by means of the sensomics concept. J. Agric. Food Chem. 2013, 61, 9583-9593. [CrossRef] [PubMed]

43. Luigi, P.; Peter, S. Characterization of the key aroma compounds in an American Bourbon whisky by quantitative measurements, aroma recombination, and omission studies. J. Agric. Food Chem. 2002, 56, 5820-5826. [CrossRef] 